\title{
The NO-cGMP-PKG signaling pathway regulates synaptic plasticity and fear memory consolidation in the lateral amygdala via activation of ERK/MAP kinase
}

\author{
Kristie T. Ota, ${ }^{1}$ Vicki J. Pierre, ${ }^{1}$ Jonathan E. Ploski, ${ }^{1}$ Kaila Queen, ${ }^{1}$ \\ and Glenn E. Schafe ${ }^{1-3}$ \\ ${ }^{7}$ Department of Psychology, Yale University, New Haven, Connecticut 06520, USA; ${ }^{2}$ Interdepartmental Neuroscience Program, \\ Yale University, New Haven, Connecticut 06520, USA
}

\begin{abstract}
Recent studies have shown that nitric oxide (NO) signaling plays a crucial role in memory consolidation of Pavlovian fear conditioning and in synaptic plasticity in the lateral amygdala (LA). In the present experiments, we examined the role of the cGMP-dependent protein kinase (PKG), a downstream effector of NO, in fear memory consolidation and long-term potentiation (LTP) at thalamic and cortical input pathways to the LA. In behavioral experiments, rats given intra-LA infusions of either the PKG inhibitor Rp-8-Br-PET-cGMPS or the PKG activator 8-Br-cGMP exhibited dose-dependent impairments or enhancements of fear memory consolidation, respectively. In slice electrophysiology experiments, bath application of Rp-8-Br-PET-cGMPS or the guanylyl cyclase inhibitor LY83583 impaired LTP at thalamic, but not cortical inputs to the LA, while bath application of 8-Br-cGMP or the guanylyl cyclase activator YC-1 resulted in enhanced LTP at thalamic inputs to the LA. Interestingly, YC-1-induced enhancement of LTP in the LA was reversed by concurrent application of the MEK inhibitor U0126, suggesting that the NO-cGMP-PKG signaling pathway may promote synaptic plasticity and fear memory formation in the LA, in part by activating the ERK/MAPK signaling cascade. As a test of this hypothesis, we next showed that rats given intra-LA infusion of the PKG inhibitor Rp-8-Br-PET-cGMPS or the PKG activator 8-Br-cGMP exhibit impaired or enhanced activation, respectively, of ERK/MAPK in the LA after fear conditioning. Collectively, our findings suggest that an NO-cGMP-PKG-dependent form of synaptic plasticity at thalamic input synapses to the LA may underlie memory consolidation of Pavlovian fear conditioning, in part, via activation of the ERK/MAPK signaling cascade.
\end{abstract}

Nitric oxide (NO) signaling has been widely implicated in synaptic plasticity and memory formation (Schuman and Madison 1991; Bredt and Snyder 1992; Chapman et al. 1992; Bohme et al. 1993; Zhuo et al. 1994; Bernabeu et al. 1995; Arancio et al. 1996; Doyle et al. 1996; Holscher et al. 1996; Suzuki et al. 1996; Son et al. 1998; Zou et al. 1998; Ko and Kelly 1999; Lu et al. 1999). A highly soluble gas generated by the conversion of L-arginine to L-citrulline by the $\mathrm{Ca}^{2+}$-regulated enzyme nitric oxide synthase (NOS), NO is known to have a variety of effects both pre- and postsynaptically. One immediate downstream effector of $\mathrm{NO}$, for example, is soluble guanylyl cyclase (sGC) (Bredt and Snyder 1992; Son et al. 1998; Denninger and Marletta 1999; Arancio et al. 2001). This enzyme directly leads to the formation of cyclicGMP, and in turn, to the activation of the cGMP-dependent protein kinase (PKG). PKG, in turn, can have a number of effects, including targeting and mobilization of synaptic vesicles in the presynaptic cell, leading to enhanced transmitter release (Hawkins et al. 1993, 1998) and also to activation of protein kinase signaling cascades in the postsynaptic cell, leading to activation of transcription and translation that are critical for long-term synaptic plasticity and memory formation (Lu et al. 1999; Chien et al. 2003).

While most widely studied in the hippocampus (Chapman et al. 1992; Bohme et al. 1993; Bernabeu et al. 1995, 1996, 1997;

\footnotetext{
${ }^{3}$ Corresponding author.
}

E-mail glenn.schafe@yale.edu; fax (203) 432-7172.

Article is online at http://www.learnmem.org/cgi/doi/10.1101/lm.1114808.
Holscher et al. 1996; Suzuki et al. 1996; Zou et al. 1998) and cerebellum (Chapman et al. 1992), recent evidence from our laboratory has suggested that NO signaling in the lateral nucleus of the amygdala (LA) is also critical to fear memory formation (Schafe et al. 2005a). In our study, neuronal NOS (nNOS) was shown to be expressed in LA neurons and in postsynaptic sites of excitatory synapses in the LA. Further, pharmacological manipulation of NO signaling in the LA using either a NOS inhibitor or a membrane-impermeable scavenger of NO impaired memory consolidation of auditory fear conditioning and LTP at auditory thalamic input synapses to the LA, in vitro (Schafe et al. 2005a). These findings suggest a role for NO signaling in Pavlovian fear conditioning and synaptic plasticity in the LA. Further, given that previous studies have failed to find effects of NOS blockade on LTP at cortical inputs to the LA (Watanabe et al. 1995), our recent findings suggest a rather specific role for $\mathrm{NO}$ signaling in synaptic plasticity at thalamic inputs to the LA.

The present study was aimed at further characterizing the role of the NO signaling pathway in fear memory consolidation and associated synaptic plasticity in the LA. In the first series of experiments, we examined the involvement of downstream effectors of NO signaling, including sGC and PKG, using pharmacological agents in both behavioral and in vitro electrophysiological experiments that both inhibit and promote activation of the NOcGMP-PKG signaling pathway. In the second series of experiments, we examined whether the NO-cGMP-PKG signaling pathway might play a unique role in synaptic plasticity at thalamic input synapses to the LA. Finally, we asked whether NO signaling in the 
LA might promote synaptic plasticity and memory formation by activating the extracellular signal-regulated kinase/mitogenactivated protein kinase (ERK/MAPK), a signaling cascade known to play a critical role in fear memory consolidation (Atkins et al. 1998; Schafe et al. 2000).

\section{Results}

\section{Inhibition of PKG in the lateral amygdala impairs fear memory consolidation and long-term potentiation at thalamic inputs to the LA}

We have recently shown that blockade of NO signaling in the LA using the NOS inhibitor 7-Nitroindazole (7-Ni) or the membrane-impermeable NO scavenger carboxy-PTIO (c-PTIO) impairs fear memory consolidation and LTP at thalamic inputs to the LA (Schafe et al. 2005a). In this first series of experiments, we examined the role of downstream targets of NO signaling in memory consolidation of auditory fear conditioning and synaptic plasticity at thalamic inputs to the LA. In behavioral experiments, we gave rats intra-LA infusion of vehicle $(0.5 \mu \mathrm{L} 0.9 \% \mathrm{NaCl})$ or of different doses of the PKG inhibitor Rp-8-Br-PET-cGMPS (0.1 or $1.0 \mu \mathrm{g} / \mathrm{side} ; 0.5 \mu \mathrm{L}$ ) prior to auditory fear conditioning and examined retention at $1,3,6$, and $24 \mathrm{~h}$ after training. In slice electrophysiology experiments, we used a combination of field and whole-cell recordings to examine the role of PKG and sGC, its upstream regulator, in LTP at thalamo-LA synapses.

\section{Behavioral experiments}

The findings of the behavioral experiment can be observed in Figure 1. Rats infused with either dose of Rp-8-Br-PET-cGMPS exhibited intact post-shock freezing during training that did not significantly differ from vehicle-infused controls (Fig. 1B). The ANOVA for post-shock freezing scores showed only a significant effect of trial $\left(F_{(2,46)}=14.91, P<0.001\right)$; the effect for drug $\left(F_{(2,23)}=2.50\right)$ and the drug by trial interaction $\left(F_{(4,46)}=1.37\right)$ were not significant. Similarly, Rp-8-Br-PET-cGMPS-infused rats tested for auditory fear memory $1 \mathrm{~h}$ following conditioning were found to have intact short-term memory (Fig. 1C). The ANOVA for fear memory at $1 \mathrm{~h}$ did not reveal a significant effect of drug condition $\left(F_{(2,23)}=1.32\right)$, for trial $\left(F_{(2,46)}=0.04\right)$, or for the drug by trial interaction $\left(F_{(4,46)}=0.32\right)$.

At $3 \mathrm{~h}$, however, differences began to emerge in the group treated with the highest dose of the PKG inhibitor (Fig. 1D). The ANOVA for the 3-h memory test showed a significant effect of drug condition $\left(F_{(2,23)}=3.46, P<0.05\right)$. The effect of trial $\left(F_{(2,46)}=0.30\right)$ and the drug by trial interaction $\left(F_{(4,46)}=0.94\right)$ were not significant. Similarly, at $6 \mathrm{~h}$, the group infused with the highest dose of Rp-8-Br-PET-cGMPS differed significantly from both the vehicle and low-dose conditions (Fig. 1E). The ANOVA for the 6-h test revealed a significant effect for group $\left(F_{(2,23)}=4.48, P<0.03\right)$. The effect of trial $\left(F_{(2,46)}=1.46\right)$ and the drug by trial interaction $\left(F_{(4,46)}=0.96\right)$ were not significant. This difference became even more pronounced at $24 \mathrm{~h}$ after training, with the ANOVA revealing a significant effect of drug condition $\left(F_{(2,23)}=5.46, P<0.01\right)$ and for trial $\left(F_{(9,207)}=4.85, P<0.01\right)$. The drug by trial interaction $\left(F_{(18,207)}=0.46\right)$ was not significant. Duncan's post-hoc $t$-tests revealed that the high-dose group differed significantly from the vehicle group on the first six trials of the LTM test $(P<0.05)$. The low-dose group failed to differ significantly from the vehicle group on any trial $(P>0.05)$.

To further examine the consolidation deficit in rats infused with Rp-8-Br-PET-cGMPS, we expressed each animal's freezing score at $24 \mathrm{~h}$ (LTM test) as a percentage of that during the $1 \mathrm{~h}$ (STM) test (Fig. 1G). The ANOVA revealed a significant difference
$\left(F_{(2,23)}=5.47, P<0.02\right)$, with Duncan's post-hoc $t$-tests revealing a significant group difference between the high-dose condition and both the low-dose and vehicle conditions $(P<0.05)$.

Our initial experiments showed that infusion of the PKG inhibitor Rp-8-Br-PET-cGMPS into the LA dose-dependently impairs memory consolidation of auditory fear conditioning. The observation of intact post-shock freezing and memory during the 1-h test (Fig. 1B,C) rules out the possibility that Rp-8-Br-PETcGMPS may have nonspecifically interfered with tone or shock processing during the training session. However, it remains possible that the memory impairment observed at $24 \mathrm{~h}$ (Fig. 1F) could reflect state-dependent learning or nonspecific damage to the LA that emerges slowly after infusion. To address these concerns, rats were reinfused with vehicle or their respective dose of drug the day following the 24-h test and retested for fear memory $1 \mathrm{~h}$ after infusion (Fig. 1H, top). Thus, these animals were tested for auditory fear memory retention under the influence of the drug. While not reaching significance $\left(F_{(2,21)}=2.93, P<0.08\right)$, perhaps due to extinction in the vehicle group, the pattern of results was nonetheless similar to that of the 24-h test, in which the drug appeared to dose-dependently impair consolidation of the memory. Thus, it is unlikely that the memory impairment observed during the original 24 -h test can be attributable to statedependent learning.

To rule out the possibility that infusion of Rp-8-Br-PETcGMPS produces delayed, yet nonspecific damage to the LA, all rats were retrained in the absence of any drug infusion $\sim 24 \mathrm{~h}$ after the end of the state-dependent test. One hour after training, animals were tested for retention of auditory fear memory (Fig. 1H, bottom). The findings revealed that all groups displayed robust and equivalent levels of freezing $\left(F_{(2,23)}=0.207\right)$, indicating that the LA was intact and not damaged by infusion of the drug. Collectively, these findings suggest that animals that had previously performed poorly on the memory-retention test likely did so due to impaired fear memory consolidation, and not because of nonspecific factors such as state-dependent learning or damage to the LA.

In our initial behavioral experiments, we infused rats with multiple doses of Rp-8-Br-PET-cGMPS prior to fear conditioning and tested each animal for retention of auditory fear conditioning at $1,3,6$, and $24 \mathrm{~h}$ following training. Since rats in our first experiment were tested multiple times across a 24 -h period, it might be argued that the memory impairments observed at 6 and $24 \mathrm{~h}$ reflect enhanced extinction under the influence of Rp-8-BrPET-cGMPS rather than impaired memory consolidation. Alternatively, since in our initial experiments the drug was likely still present in the LA during the 1-h memory test, it might be argued that our "consolidation" deficit observed at $24 \mathrm{~h}$ instead reflects a "reconsolidation" deficit. To examine each of these possibilities, we gave a separate group of rats intra-LA infusion of vehicle $(0.5 \mu \mathrm{L} 0.9 \% \mathrm{NaCl})$ or of different doses of Rp-8-Br-PET-cGMPS $(0.1$ or $1.0 \mu \mathrm{g} / \mathrm{side} ; 0.5 \mu \mathrm{L})$ prior to auditory fear conditioning and examined retention at $24 \mathrm{~h}$ after training without intervening memory tests (Fig. 2A). Rats infused with either dose of Rp-8-Br-PET-cGMPS exhibited intact post-shock freezing during training that did not significantly differ from vehicle-infused controls (Fig. 2B). The ANOVA for post-shock freezing scores showed only a significant effect of trial $\left(F_{(1,15)}=46.46\right.$, $P<0.001)$; the effect for $\operatorname{drug}\left(F_{(2,15)}=0.05\right)$ and the drug by trial interaction $\left(F_{(2,15)}=0.05\right)$ were not significant. At $24 \mathrm{~h}$, however, differences between the conditions were evident for both groups treated with the PKG inhibitor (Fig. 2C). The ANOVA for the 24-h memory test showed a significant effect of drug condition $\left(F_{(2,15)}=3.75, P<0.05\right)$ and for trial $\left(F_{(9,135)}=1.96, P<0.05\right)$. The drug by trial interaction $\left(F_{(18,135)}=0.46\right)$ was not significant. Duncan's post-hoc $t$-tests revealed that the high-dose group 


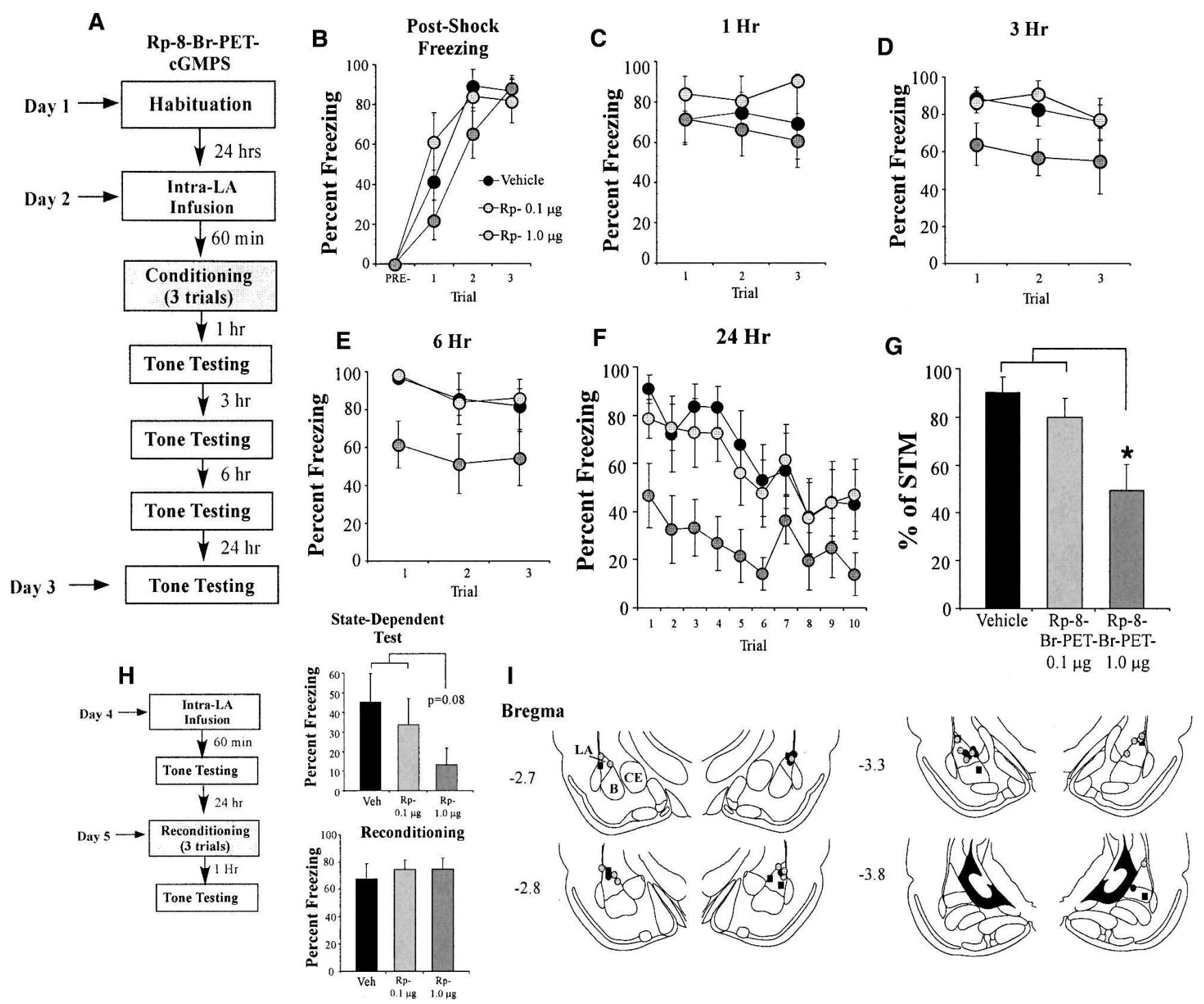

Figure 1. Inhibition of PKG in the LA impairs auditory fear memory consolidation. (A) Schematic of the behavioral protocol. Rats were given intra-LA infusion of either the vehicle or a high $(1.0 \mu \mathrm{g})$ or low $(0.1 \mu \mathrm{g})$ dose of Rp-8-Br-PET-cGMPS. Sixty minutes later they were trained with three tone-shock pairings, then tested for retention of auditory fear conditioning at 1, 3, 6, and $24 \mathrm{~h}$ following conditioning. (B) Mean ( \pm SEM) post-shock freezing between conditioning trials in rats given intra-LA infusions of $0.15 \mathrm{M} \mathrm{NaCl}$ (vehicle; $n=8), 1.0 \mu \mathrm{g} \mathrm{Rp}-8$-Br-PET-cGMPS $(n=8)$, or $0.1 \mu \mathrm{g} \mathrm{Rp}-8-\mathrm{Br}$-PETcGMPS $(n=8)$. (C) Mean ( \pm SEM) auditory fear memory assessed at $1 \mathrm{~h}$ following conditioning. (D) Mean $( \pm$ SEM) auditory fear memory assessed at $3 \mathrm{~h}$ following conditioning. ( $E$ ) Mean ( \pm SEM) auditory fear memory assessed at $6 \mathrm{~h}$ following conditioning. $(F)$ Mean ( \pm SEM) auditory fear memory assessed at $24 \mathrm{~h}$ following conditioning. (G) LTM expressed as a percentage of STM in each group. Each rat's 24-h memory score is expressed as a percentage of its 1-h memory score. $(H)$ Schematic of the state-dependency and reconditioning trials. Rats were given intra-LA infusion of their original drug dose $(0.15 \mathrm{M} \mathrm{NaCl}$ [vehicle; $n=8$ ], $1.0 \mu \mathrm{g} \mathrm{Rp-8-Br-PET-cGMPS} \mathrm{[} n=8$ ], or $0.1 \mu \mathrm{g} \mathrm{Rp-8-Br-PET-cGMPS}[n=8]$ ), then tested for auditory fear conditioning $1 \mathrm{~h}$ later. Rats were then reconditioned drug free and tested for auditory fear conditioning $1 \mathrm{~h}$ later. Mean ( \pm SEM) auditory fear memory assessed at $1 \mathrm{~h}$ following state-dependent test and at $1 \mathrm{~h}$ following reconditioning. (I) Histological verification of cannula placements for rats infused with $1.0 \mu \mathrm{g} \mathrm{Rp-8-Br-PET-cGMPS} \mathrm{(gray} \mathrm{circles),} 0.1 \mu \mathrm{g} \mathrm{Rp-8-Br-PET-cGMPS} \mathrm{(black} \mathrm{circles),} \mathrm{or} 0.15 \mathrm{M} \mathrm{NaCl}$ vehicle (black squares). Panels adapted from Paxinos and Watson (1997). $\left(^{*}\right) P<0.05$ relative to vehicle and $0.1 \mu \mathrm{g}$ Rp-8-Br-PET-cGMPS.

differed significantly from the vehicle group on all trials of the LTM test with the exception of trials 3,8 , and $10(P<0.05)$. The low-dose group, in contrast, differed from the vehicle group only on trials 2 and $4(P<0.05)$. Unlike in the first experiment, the high- and low-dose groups did not differ from each other on any trial $(P>0.05)$, indicating that the low dose of Rp-8-Br-PETcGMPS was more effective at impairing fear memory in this experiment. This finding is likely due to the fact that a slightly weaker training protocol was used for this experiment (one pairing vs. three pairings). Overall, however, these findings suggest that it is unlikely that infusion of Rp-8-Br-PET-cGMPS in our previous experiments impaired memory by enhancing fear extinction. It is also unlikely that our initial findings are solely attributable to a "reconsolidation" effect. Rather, our findings are consistent with the interpretation that signaling via the NO-cGMP-PKG pathway is critical for fear memory consolidation.

Histological verification of the cannula placements for rats infused with Rp-8-Br-PET-cGMPS in each behavioral experiment is presented in Figures 1I and 2D. Cannula tips were observed to lie throughout the LA at various rostro-caudal levels. Only rats with cannula tips at or within the boundaries of the LA or in the adjacent basal nucleus were included in the data analysis. 

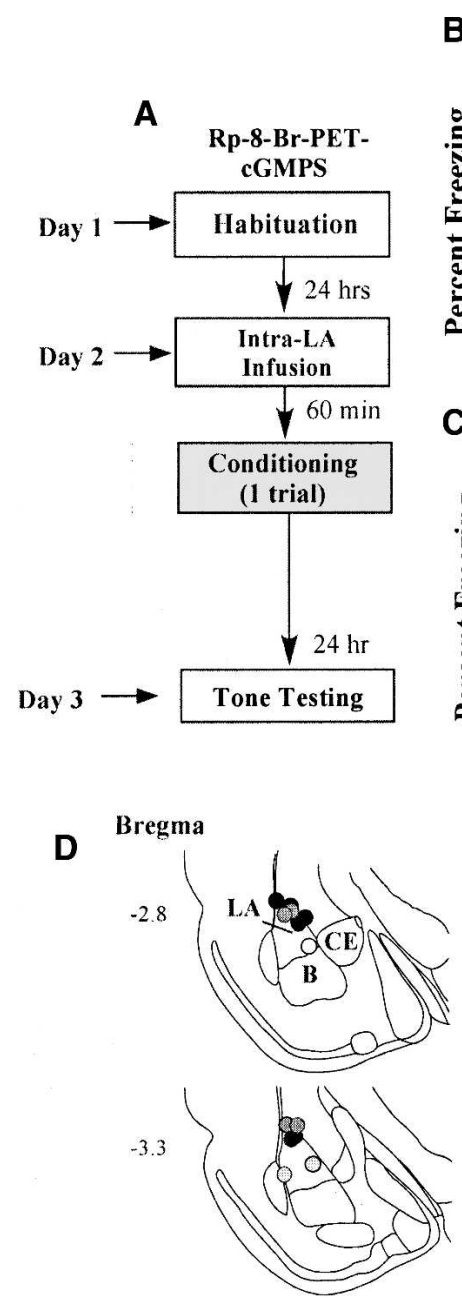

B
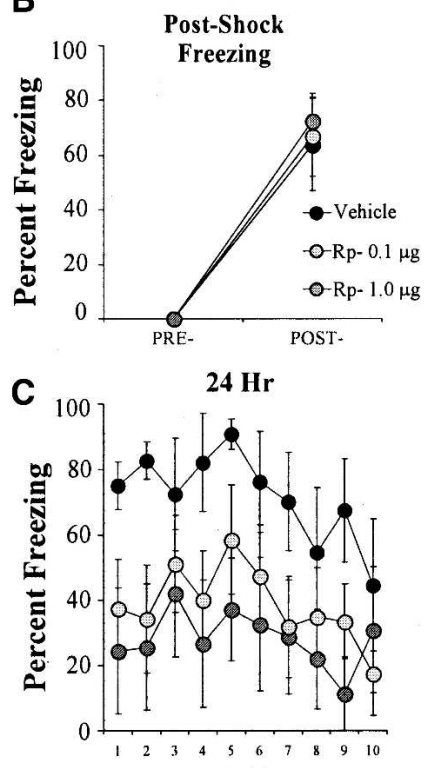

Trial

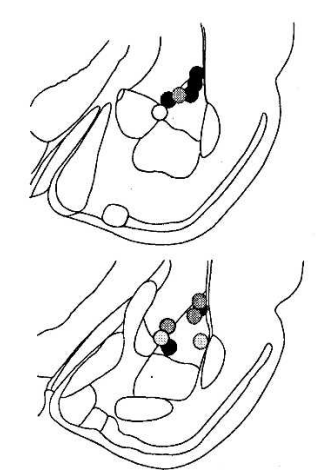

Figure 2. Inhibition of PKG in the LA impairs auditory fear memory consolidation independent of affecting fear extinction or reconsolidation. (A) Schematic of the behavioral protocol. Rats were given intra-LA infusion of either the vehicle or a high $(1.0 \mu \mathrm{g})$ or low $(0.1 \mu \mathrm{g})$ dose of $\mathrm{Rp}-8$-Br-PET-cGMPS. Sixty minutes later they were trained with a single tone-shock pairing, then tested for retention of auditory fear conditioning $24 \mathrm{~h}$ later. $(B)$ Mean $( \pm$ SEM) post-shock freezing in rats given intra-LA infusions of $0.15 \mathrm{M} \mathrm{NaCl}$ (vehicle; $n=6$ ), $1.0 \mu \mathrm{g} \mathrm{Rp-8-Br-PET-cGMPS}$ $(n=5)$, or $0.1 \mu \mathrm{g} \mathrm{Rp}-8-\mathrm{Br}-\mathrm{PET}-\mathrm{cGMPS}(n=7)$. (C) Mean ( \pm SEM) auditory fear memory assessed at $24 \mathrm{~h}$ following conditioning. $(D)$ Histological verification of cannula placements for rats infused with $1.0 \mu \mathrm{g} \mathrm{Rp}-8-\mathrm{Br}$ PET-cGMPS (gray circles), $0.1 \mu \mathrm{g}$ Rp-8-Br-PET-cGMPS (light-gray circles), or $0.15 \mathrm{M} \mathrm{NaCl}$ vehicle (black circles). Panels adapted from Paxinos and Watson (1997).

\section{Slice electrophysiology}

We next examined the role of sGC-PKG signaling in synaptic plasticity at thalamic inputs to the LA using slice-recording methods (Fig. 3A). In these initial experiments, we examined this question using both field recordings and whole-cell patch clamp techniques. In our field-recording experiments, we bath applied either the PKG inhibitor Rp-8-Br-PET-cGMPS $(1 \mu \mathrm{M})$ or the sGC inhibitor LY83583 $(5 \mu \mathrm{M})$ to the slice prior to inducing LTP with a $100-\mathrm{Hz}$ tetanus (given three times, separated by $1 \mathrm{~min}$ at $50 \%$ higher stimulation intensity; see Materials and Methods for details). This low concentration of Rp-8-Br-PET-cGMPS has been shown to be highly specific to PKG and to have little effect on other kinases such as PKA (Lu et al. 1999).

In our whole-cell experiments, we examined the effect of bath application of $1 \mu \mathrm{M}$ Rp-8-Br-PET-cGMPS prior to delivery of a 30-Hz tetanus (Schafe et al. 2005a). In separate whole-cell experiments, we next examined the effect of delivering $\mathrm{Rp}-8-\mathrm{Br}$ PET-cGMPS $(250 \mu \mathrm{M})$ to the cell in the patch pipette. This latter experiment allowed us to restrict the delivery of Rp-8-Br-PETcGMPS to the postsynaptic cell to determine whether signaling via PKG might play a role in postsynaptic aspects of plasticity at thalamo-LA synapses.

For each experiment, the effects of bath application of each compound on the amplitude or slope of the evoked response was also measured for a $20-30 \mathrm{~min}$ period prior to LTP induction to determine whether the drugs had any effect on routine transmission at thalamo-LA synapses.

Relative to ACSF-perfused slices, Rp-8-Br-PET-cGMPS was found to inhibit LTP induced by stimulation of thalamic inputs to the LA (Fig. 3B). The control slices showed $125.28 \pm 11.17 \%$ potentiation, which was significantly different from baseline $\left(t_{(6)}=2.29, P<0.05\right.$; one-tailed). On the other hand, Rp-8-BrPET-cGMPS-treated slices showed only $93.59 \pm 7.88 \%$ potentiation, which was not significantly different from baseline $\left(t_{(5)}=0.96\right)$. A comparison of the control and inhibitor-treated slices revealed significantly higher levels of potentiation in the ACSF slices than in the PKG inhibitor-treated slices $\left(t_{(11)}=2.24\right.$, $P<0.05)$. Importantly, bath application of Rp-8-Br-PET-cGMPS had no effect on baseline transmission alone $\left(t_{(5)}=0.47\right.$; Fig. $3 \mathrm{~B}$, inset). We also found that inhibition of sGC impaired thalamoLA LTP. Relative to ACSF-perfused controls, bath application of LY83583 impaired LTP (Fig. 3C). The control (ACSF-treated) slices exhibited $120.97 \pm 6.23 \%$ potentiation, which was significantly different from baseline levels $\left(t_{(12)}=3.60, P<0.01\right)$, whereas inhibitor-treated slices showed only $103.13 \pm 6.66 \%$ potentiation, which was not significantly different from baseline $\left(t_{(7)}=0.60\right)$. Further, the control slices and the inhibitor-treated slices exhibited significantly different levels of LTP from each other $\left(t_{(19)}=1.87, P<0.05\right.$; one-tailed). There was no effect, however, of bath application of LY83583 on routine transmission alone $\left(t_{(5)}=1.44\right.$; Fig. 3C, inset).

The findings of the whole-cell experiments mirrored those of the field recordings. Results showed that bath application of Rp-8-Br-PET-cGMPS impaired LTP at thalamic inputs to the LA (Fig. 3D). The control cells showed $126.78 \pm 6.48 \%$ potentiation, which was significantly higher than baseline $t_{(7)}=4.09$, $P<0.01)$. In contrast, the PKG inhibitor-treated cells showed $90.28 \pm 7.71 \%$ potentiation, which was not significantly different from baseline $\left(t_{(6)}=0.82\right)$, but was significantly different from the ACSF-treated cells $\left(t_{(13)}=3.65, P<0.01\right)$. Also, as before, bath application of the drug alone had no effect on routine transmission $\left(t_{(5)}=0.29\right.$; Fig. 3D, inset). A similar picture emerged when Rp-8-Br-PET-cGMPS was dissolved in the recording pipette (Fig. 3E). The control cells showed $123.72 \pm 6.85 \%$ potentiation, which was significantly higher than baseline $\left(t_{(13)}=3.52\right.$, $P<0.01)$. In contrast, the PKG inhibitor-treated cells showed $86.93 \pm 7.6 \%$ potentiation, which was not significantly different from baseline $\left(t_{(4)}=1.84\right)$, but was significantly different from controls cells $\left(t_{(17)}=2.95, P<0.01\right)$. Further, resting membrane potentials $(\mathrm{Vm})$ remained constant throughout the experiment in cells treated with Rp-8-Br-PET-cGMPS in the pipette. An analysis comparing $\mathrm{Vm}$ at the beginning (just after patching) and at the end of the experiment for each cell revealed no significant effects $(\mathrm{Vm}$ beginning $=60 \pm 1.07 \% ; \mathrm{Vm}$ end $=61.2 \pm 0.86 \%$; $\left.t_{(4)}=1.5, P>0.05\right)$.

Together, these findings provide support for a role of sGCcGMP-PKG signaling in LTP induced through stimulation of thalamic inputs to the LA and in fear memory consolidation. These findings extend those of our previous report (Schafe et al. 2005a) and collectively provide strong evidence that the NO-cGMP-PKG 
A
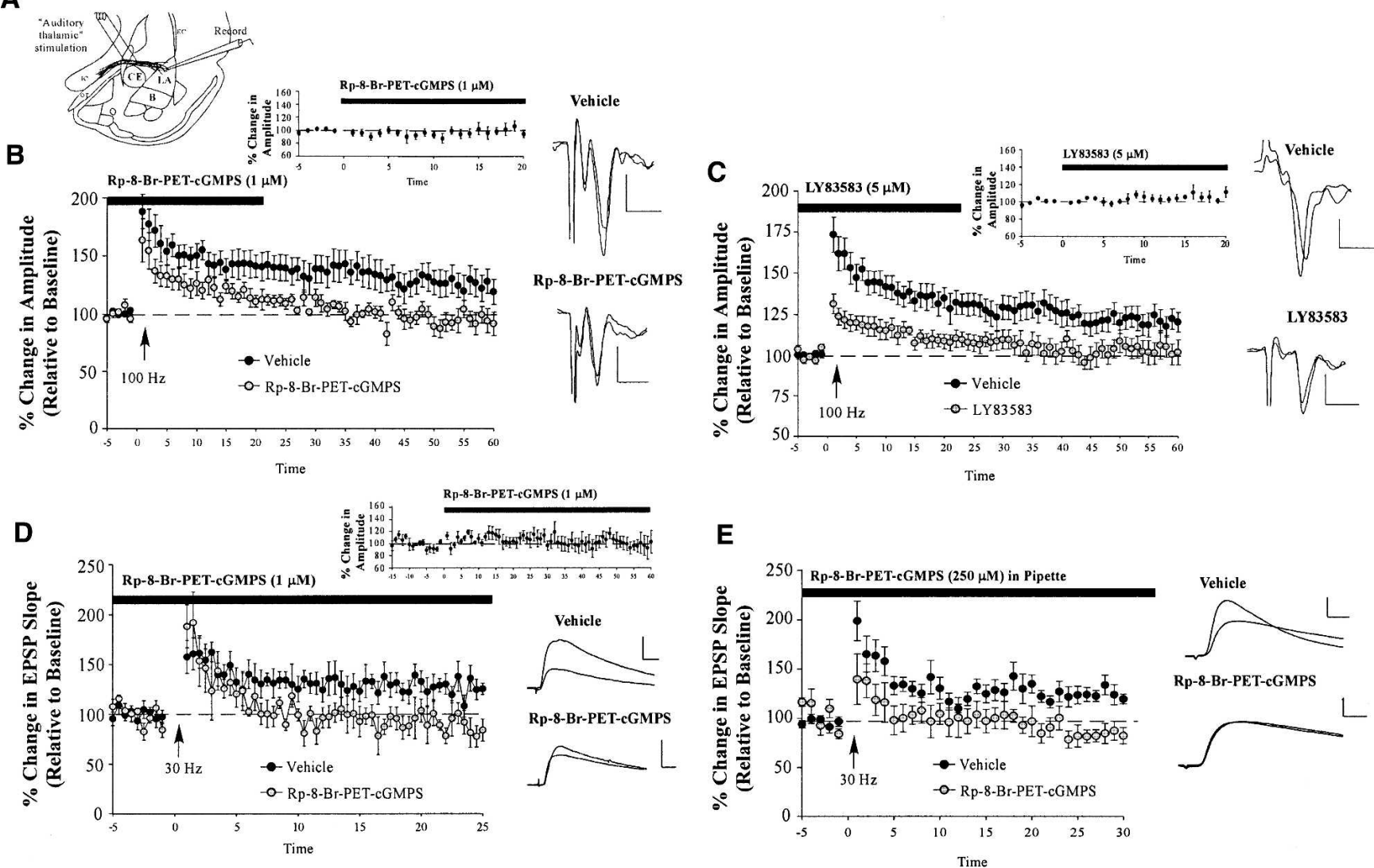

Figure 3. Inhibition of the SGC-CGMP-PKG signaling pathway impairs LTP at thalamic inputs to the LA. (A) Schematic of the amygdala slice preparation for "thalamic" stimulation, showing placement of stimulating and recording electrodes. Recordings were made just below the site of termination of auditory thalamic fibers terminating in the LA. (IC) Internal capsule; (OT) optic tract; (EC) external capsule. (B) Mean ( \pm SEM) percentage field potential amplitude (relative to baseline) in slices treated with vehicle $(n=7$; black circles) or $1 \mu \mathrm{M}$ Rp-8-Br-PET-cGMPS $(n=6$; gray circles). Traces from an individual experiment before and 50 min following tetanic stimulation and transmission following 20 min of bath application of drug are shown in the inset. Scale, $0.2 \mathrm{mV}$ by $10 \mathrm{msec}$. (C) Mean ( \pm SEM) percentage field potential amplitude (relative to baseline) in slices treated with vehicle $(n=13$; black circles) or $5 \mu \mathrm{M}$ LY83583 ( $n=8$; gray circles). Traces from an individual experiment before and 50 min following tetanic stimulation and transmission following $20 \mathrm{~min}$ of bath application of drug are shown in the inset. Scale, $0.2 \mathrm{mV}$ by $10 \mathrm{msec}$. (D) Mean ( \pm SEM) percentage EPSP slope (relative to baseline) in slices treated with vehicle ( $n=8$; black circles) or $1 \mu \mathrm{M} \mathrm{Rp-8-Br-PET-cGMPS} \mathrm{(} n=7$; gray circles). Traces from an individual experiment before and $50 \mathrm{~min}$ following tetanic stimulation and transmission following $60 \mathrm{~min}$ of bath application of drug are shown in the inset. Scale, $5 \mathrm{mV}$ by $10 \mathrm{msec}$. ( $E$ ) Mean ( \pm SEM) percentage EPSP slope (relative to baseline) in cells treated with vehicle $(n=14$; black circles) or $250 \mu \mathrm{M}$ Rp-8-Br-PET-cGMPS $(n=5$; gray circles) in the recording pipette. Traces from an individual experiment before and 50 min following tetanic stimulation are shown in the inset. Scale, $5 \mathrm{mV}$ by $10 \mathrm{msec}$.

signaling pathway is required for fear memory consolidation and synaptic plasticity in the LA.

\section{Inhibition of NO-cGMP-PKG signaling fails to impair long-term potentiation at cortical inputs to the LA} In the previous series of experiments, we showed that inhibition of PKG, a downstream substrate of NO signaling, impairs fear memory consolidation and synaptic plasticity at thalamic input synapses to the LA. A previous study, however, failed to find effects of inhibition of NO signaling on amygdala LTP induced at cortical inputs (Watanabe et al. 1995). This pattern of findings raises the intriguing possibility that LTP at thalamic and cortical inputs to the LA might be characterized by distinct biochemical mechanisms. To further evaluate this possibility, we next examined the effect of inhibition of NO-cGMP-PKG signaling on LTP at cortical inputs to the LA (Fig. 4A). Given that we have not previously studied LTP at cortical-LA synapses, we began by asking whether our $100-\mathrm{Hz}$ LTP induction protocol induces LTP at cortical-LA inputs that is sensitive to NMDAR blockade with AP-5 $(50 \mu \mathrm{M})$, as has been reported in other studies (Huang and Kandel
1998). Next, we examined the effects of the NOS inhibitor 7-Nitroindazole (7-Ni; $30 \mu \mathrm{M})$, the sGC inhibitor LY83583 $(5 \mu \mathrm{M})$, and the PKG inhibitor Rp-8-Br-PET-cGMPS $(1 \mu \mathrm{M})$ on LTP at cortico-LA synapses.

Our $100-\mathrm{Hz}$ protocol successfully induced LTP at cortical inputs to the LA that was significantly impaired by bath application of AP-5 (Fig. 4B). Control slices exhibited $130.77 \pm 9.40 \%$ potentiation, which differed significantly from baseline levels $\left(t_{(7)}=3.31, P<0.05\right)$. In contrast, slices perfused with AP-5 failed to exhibit LTP, with potentiation levels remaining at $109.53 \pm 6.35 \%$, which did not differ significantly from baseline levels $\left(t_{(8)}=1.64\right)$. Further, control and AP-5-treated slices differed significantly from each other $\left(t_{(15)}=1.91 ; P<0.05\right.$; onetailed).

In contrast to slices treated with AP-5, slices treated with 7-Ni, LY83583, or Rp-8-Br-PET-cGMPS exhibited intact LTP at cortical inputs (Fig. 4B-D). In the 7-Ni experiment, control slices potentiated to $130.77 \pm 9.40 \%$, which differed significantly from baseline levels $\left(t_{(7)}=3.31, P<0.05\right)$. Slices perfused with 7 -Ni potentiated similarly to $129.51 \pm 12.55 \%$, which also differed significantly from baseline levels $\left(t_{(10)}=2.27, P<0.05\right)$. 


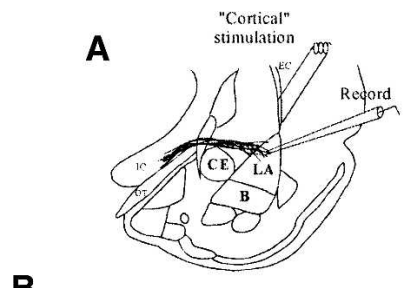

B

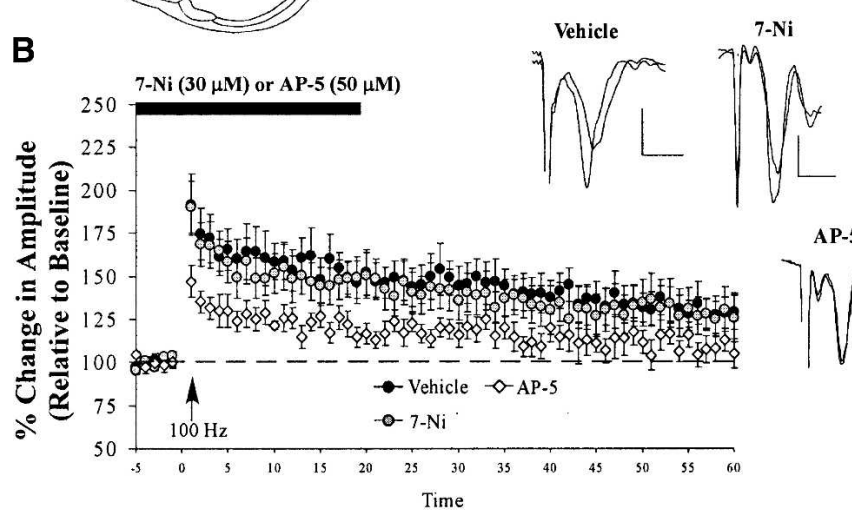

C
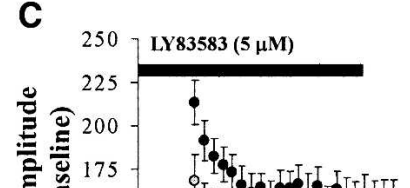

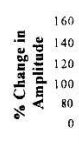
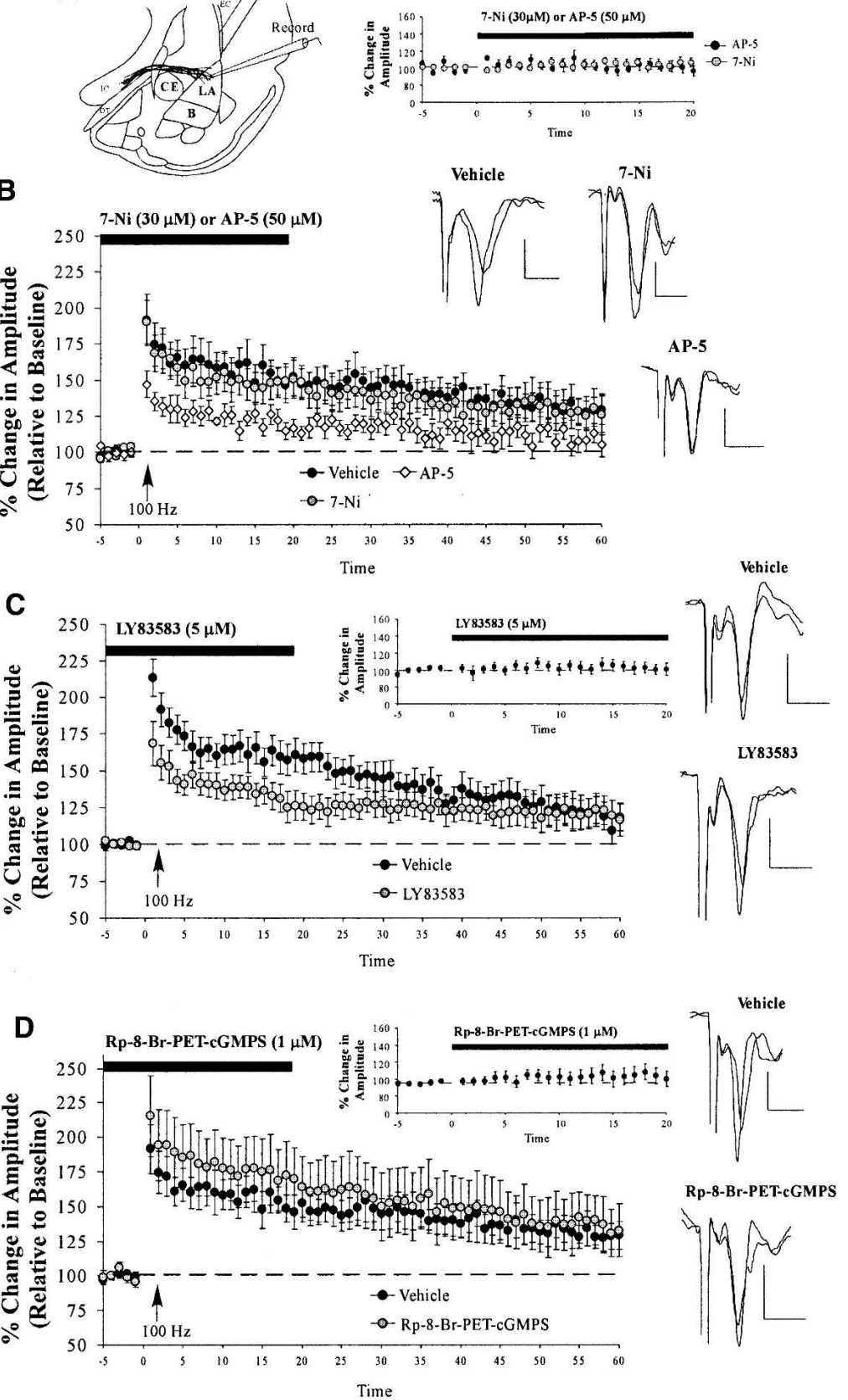

Tim

Figure 4. LTP at cortical inputs is unaffected by inhibition of the NO-cGMP-PKG signaling pathway. (A) Schematic of the amygdala slice preparation for "cortical" stimulation, showing placement of stimulating and recording electrodes. Recordings were made just below the site of termination of auditory thalamic fibers terminating in the LA. (IC) Internal capsule; (OT) optic tract; (EC) external capsule. $(B)$ Mean $( \pm$ SEM) percentage field potential amplitude (relative to baseline) in slices treated with vehicle ( $n=8$; black circles), $30 \mu \mathrm{M} 7-\mathrm{Ni}(n=11$; gray circles), or $50 \mu \mathrm{M} \mathrm{AP}-5$ ( $n=9$; white diamonds). Traces from an individual experiment before and $50 \mathrm{~min}$ following tetanic stimulation and transmission following 20 min of bath application of drug are shown in the inset. (C) Mean ( \pm SEM) percentage field potential amplitude (relative to baseline) in slices treated with vehicle $(n=11$; black circles) or $5 \mu \mathrm{M}$ LY83583 ( $n=12$; gray circles). Traces from an individual experiment before and 50 min following tetanic stimulation and transmission following $20 \mathrm{~min}$ of bath application of drug are shown in the inset. $(D)$ Mean ( \pm SEM) percentage field potential amplitude (relative to baseline) in slices treated with vehicle ( $n=8$; black circles) or $1 \mu \mathrm{M} \mathrm{Rp-8-Br-PET-cGMPS}(n=9$; gray circles). Traces from an individual experiment before and $50 \mathrm{~min}$ following tetanic stimulation and transmission following $20 \mathrm{~min}$ of bath application of drug are shown in the inset. Scale, $0.2 \mathrm{mV}$ by $10 \mathrm{msec}$.
Further statistical analyses showed that the ACSF and 7-Ni-treated slices did not differ statistically in their amount of potentiation $\left(t_{(17)}=0.07\right)$, showing no effect of the drug on cortical LTP. A similar finding was observed following bath application of the sGC inhibitor LY83583 (Fig. 4C). The control slices potentiated to $120.46 \pm 9.50 \%$, which differed significantly from baseline levels $\left(t_{(10)}=2.17\right.$; $P<0.05$; one-tailed). Similarly, LY83583-treated slices exhibited $120.88 \pm 10.32 \%$ potentiation, which differed significantly from baseline levels $\left(t_{(11)}=2.03, P<0.05\right.$; one-tailed), but not from ACSF-treated controls $\left(t_{(21)}=0.02\right)$. Finally, bath application of the PKG inhibitor Rp-8-Br-PET-cGMPS also failed to impair LTP at cortical inputs to the LA (Fig. 4D). Control slices showed $130.78 \pm 9.40 \%$ potentiation, which was significantly different from baseline $\left(t_{(7)}=3.31, P<0.05\right)$. Inhibitortreated slices behaved similarly: They showed $137.07 \pm 18.97 \%$ potentiation, which was significantly different from baseline $\left(t_{(8)}=2.02, P<0.05\right.$; one-tailed) and did not differ from control slices in their levels of potentiation $\left(t_{(15)}=0.29\right)$.

Importantly, none of the drugs used in this experiment were found to have effects on routine transmission at cortical inputs to the LA. Bath application of AP-5 (Fig. 4B, inset), 7-Ni (Fig. 4B, inset), LY83583 (Fig. 4C, inset), or Rp-8Br-PET-cGMPS (Fig. 4D, inset) all failed to affect the baseline amplitude of evoked responses (AP-5: $t_{(6)}=0.49 ; 7-\mathrm{Ni}$ : $t_{(10)}=0.63$; LY83583: $t_{(11)}=0.47 ;$ Rp-8Br-PET-cGMPS: $t_{(8)}=0.88$, respectively).

Collectively, these findings suggest that LTP at cortical inputs to the LA is not impaired by inhibitors of the NOcGMP-PKG signaling pathway. These findings replicate and extend those of a previous study (Watanabe et al. 1995) and suggest that synaptic plasticity at thalamic and cortical inputs to the LA may be subserved, in part, by distinct biochemical mechanisms.

\section{Activation of PKG in the lateral amygdala enhances fear memory consolidation and synaptic plasticity at thalamo-LA synapses} Our previous experiments relied exclusively on pharmacological inhibitors of the NO-cGMP-PKG signaling pathway. To further examine the role of PKG in fear memory formation, in this series of experiments we examined the effect of a pharmacological activator of PKG on fear memory consolidation and LTP at thalamo-LA synapses. In behavioral experiments, we gave rats intra-LA infu- 
sion of vehicle $(0.5 \mu \mathrm{L} 0.9 \% \mathrm{NaCl})$ or of different doses of the PKG activator 8 -Br-cGMP (1.0 or $10.0 \mu \mathrm{g} / \mathrm{side}$; $0.5 \mu \mathrm{L}$ ) prior to auditory conditioning, and examined retention at $1,3,6$, and 24 $\mathrm{h}$ after training (Fig. 5A). In slice electrophysiology experiments, we bath-applied 8-Br-cGMP $(1 \mu \mathrm{M})$ or the sGC activator YC-1 (1 $\mu \mathrm{M})$ to amygdala slices prior to LTP induction. In our behavioral experiments, we used a slightly weaker training protocol consisting of two tone-shock pairings, so that enhancements in fear memory could be readily observed. Similarly, in our LTP experiments, we used a protocol that does not by itself reliably induce long-lasting LTP (three 100-Hz trains, separated by $1 \mathrm{~min}$, at test stimulation intensity; see Materials and Methods for details).

\section{Behavioral experiments}

Rats infused with either dose of 8-Br-cGMP exhibited intact postshock freezing during training that did not significantly differ from that of vehicle-infused controls (Fig. 5B). The ANOVA for post-shock freezing scores showed only a significant effect of trial $\left(F_{(2,62)}=70.46, P<0.001\right)$; the effect of drug $\left(F_{(2,31)}=1.45\right)$ and the drug by trial interaction $\left(F_{(4,62)}=0.56\right)$ were not significant. Similarly, 8-Br-cGMP-infused rats tested for auditory fear memory $1 \mathrm{~h}$ following conditioning were found to have intact short-term memory (Fig. 5C). The ANOVA for fear memory at 1 $\mathrm{h}$ showed only a significant effect for trial $\left(F_{(2,62)}=4.64\right.$, $P<0.05)$. The effect for drug $\left(F_{(2,31)}=1.70\right)$ and the drug by trial interaction $\left(F_{(4,62)}=1.14\right)$ were not significant. Similarly, at $3 \mathrm{~h}$, each group exhibited equivalent retention (Fig. 5D). The ANOVA for the 3-h memory test revealed no significant effect of drug condition $\left(F_{(2,31)}=1.83\right)$, trial $\left(F_{(2,62)}=0.73\right)$, or drug-by-trial interaction $\left(F_{(4,62)}=2.41\right)$.

At $6 \mathrm{~h}$, however, a difference began to emerge in the group treated with the highest dose of the PKG activator (Fig. 5E). The
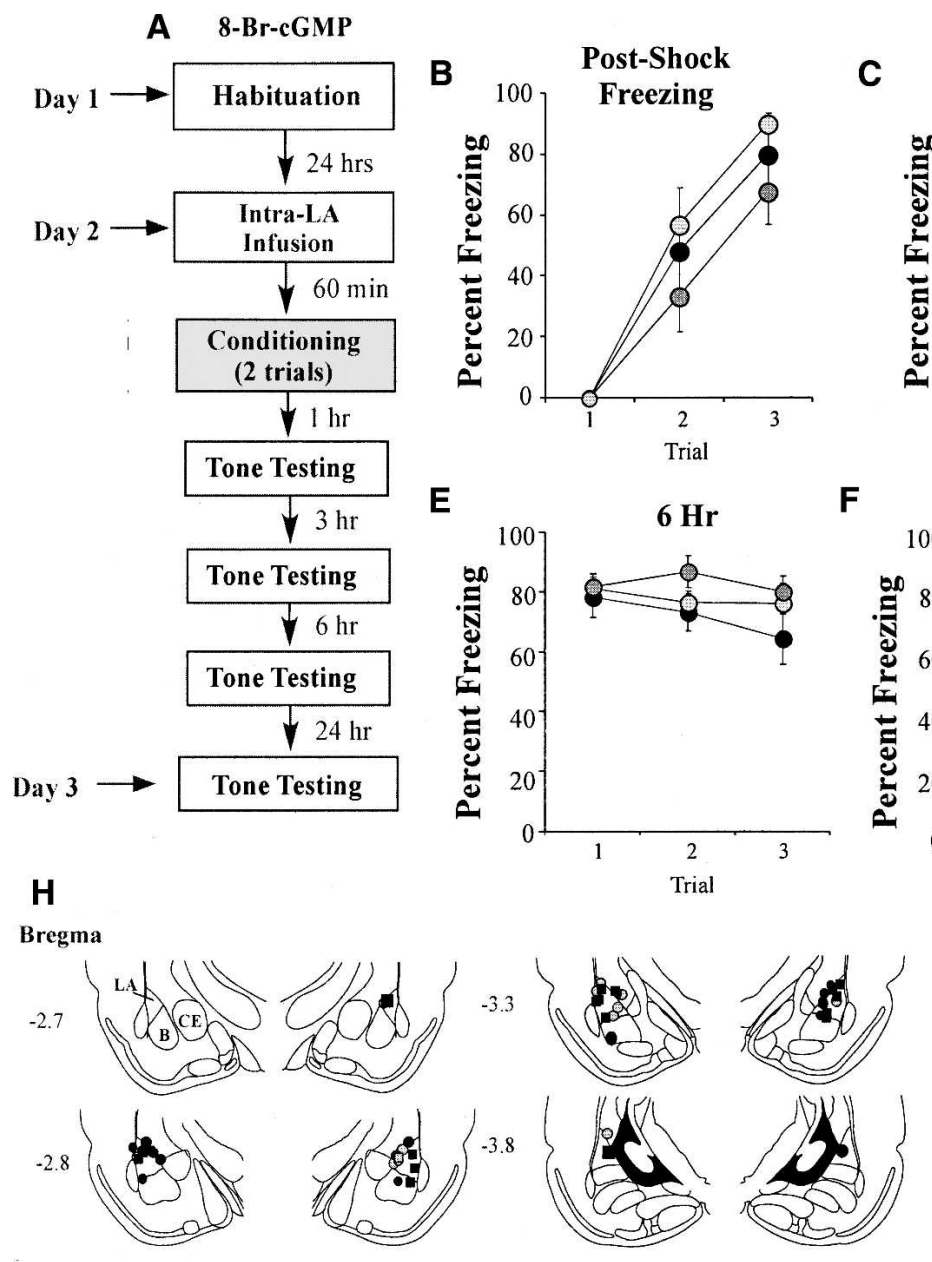

B

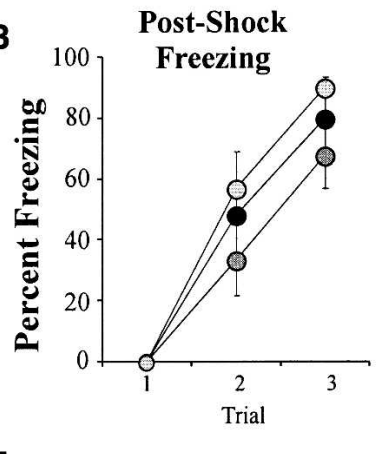

E

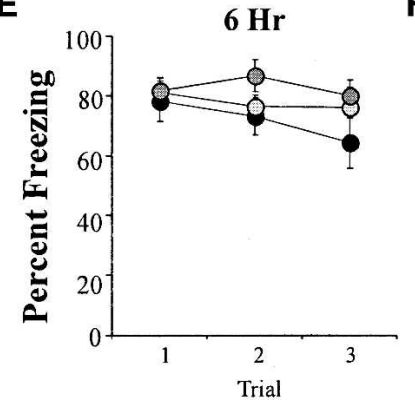

$\mathbf{F}$
C

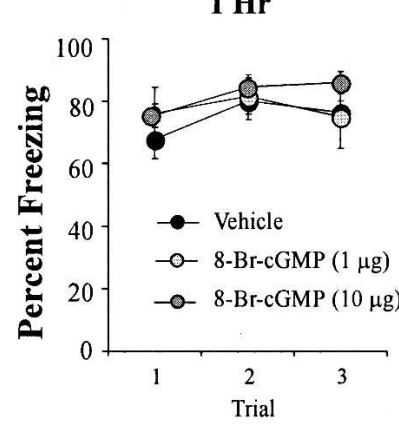

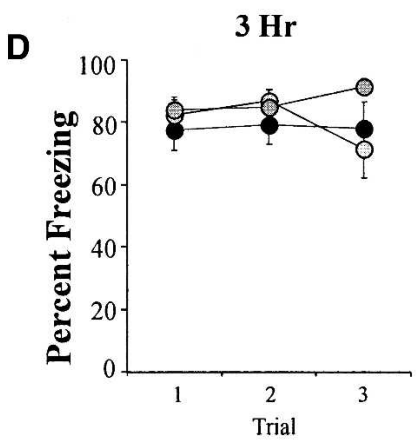
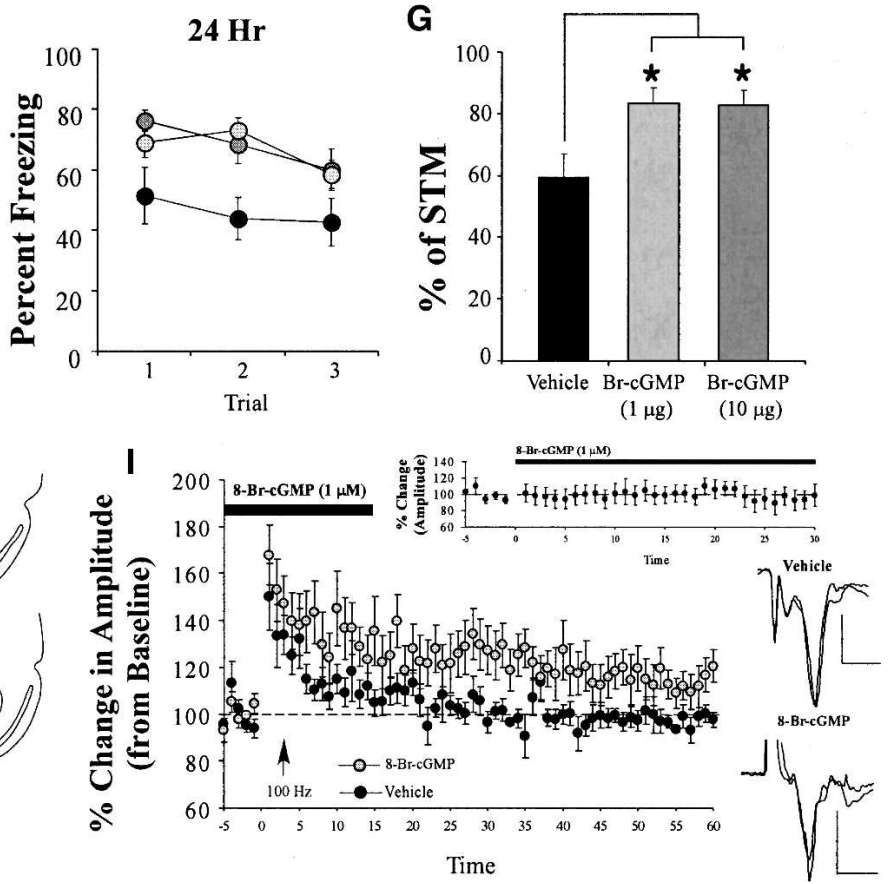

Figure 5. Activation of PKG in the LA enhances auditory fear memory consolidation. (A) Schematic of the behavioral protocol. Rats were given intra-LA infusion of the vehicle or a high $(10 \mu \mathrm{g})$ or low $(1 \mu \mathrm{g})$ dose of 8 -Br-cGMP. Sixty minutes later they were trained with two tone-shock pairings, then tested for retention of auditory fear conditioning at $1,3,6$, and $24 \mathrm{~h}$ following conditioning. (B) Mean ( \pm SEM) post-shock freezing between conditioning trials in rats given intra-LA infusions of $0.15 \mathrm{M} \mathrm{NaCl}$ (vehicle; $n=11$ ), $1 \mu \mathrm{g} 8$ - $\mathrm{Br}-\mathrm{CGMP}(n=12)$, or $10 \mu \mathrm{g} 8$ - $\mathrm{Br}$-cGMP $(n=11)$. (C) Mean ( \pm SEM) auditory fear memory assessed at $1 \mathrm{~h}$ following conditioning. (D) Mean ( \pm SEM) auditory fear memory assessed at $3 \mathrm{~h}$ following conditioning. (E) Mean $( \pm$ SEM) auditory fear memory assessed at $6 \mathrm{~h}$ following conditioning. ( $F$ ) Mean $( \pm$ SEM) auditory fear memory assessed at $24 \mathrm{~h}$ following conditioning. (G) Percentage of STM in each group. Each rat's $24-\mathrm{h}$ memory score is expressed as a percentage of its 1-h memory score. $(H)$ Histological verification of cannula placements for rats infused with $10 \mu \mathrm{g} 8$-Br-cGMP (gray circles), $1 \mu \mathrm{g} 8$-Br-cGMP (black circles), or $0.15 \mathrm{M} \mathrm{NaCl}$ vehicle (black squares). Panels adapted from Paxinos and Watson (1997). (I) Mean ( \pm SEM) percentage field potential amplitude (relative to baseline) in slices treated with vehicle ( $n=6$; black circles) or $1 \mu \mathrm{M} 8-\mathrm{Br}-\mathrm{cGMP}(n=7$; gray circles). Traces from an individual experiment before and 50 min following tetanic stimulation and transmission following $30 \mathrm{~min}$ of bath application of drug are shown in the inset. Scale, $0.2 \mathrm{mV}$ by $10 \mathrm{msec}$. $\left(^{*}\right) P<0.05$ relative to vehicle. 

drug condition $\left(F_{(2,31)}=3.76, P<0.05\right)$. The effect of trial $\left(F_{(2,62)}=3.04\right)$ and the drug by trial interaction $\left(F_{(4,62)}=2.01\right)$ were not significant. This difference became even more pronounced at $24 \mathrm{~h}$ after training (Fig. 5F), with the ANOVA revealing a significant effect of drug condition $\left(F_{(2,31)}=4.17, P<0.03\right)$ and of trial $\left(F_{(2,62)}=4.27, P<0.02\right)$. The drug by trial interaction $\left(F_{(4,62}=1.85\right)$ was not significant. Duncan's post-hoc $t$-tests revealed that both the high- and low-dose drug groups differed significantly from the vehicle group on each trial of the LTM test
ANOVA for the 6-h memory test showed a significant effect of

$(P<0.05)$. Similarly, examination of the long-term memory $(24 \mathrm{~h})$ as a function of short-term memory $(1 \mathrm{~h})$ revealed a significant group difference based on drug condition $\left(F_{(2,31)}=3.83, P<0.04\right)$, with Duncan's post-hoc $t$-tests revealing a significant difference between the vehicle control and both the high- and low-dose conditions ( $P<0.03$ for both) (Fig. 5G).

Histological verification of the cannula placements for rats infused with 8-Br-cGMP is presented in Figure 5H. Cannula tips were observed to lie throughout the LA at various rostro-caudal levels. Only rats with cannula tips at or within the boundaries of the LA or the adjacent basal nucleus were included in the data analysis.
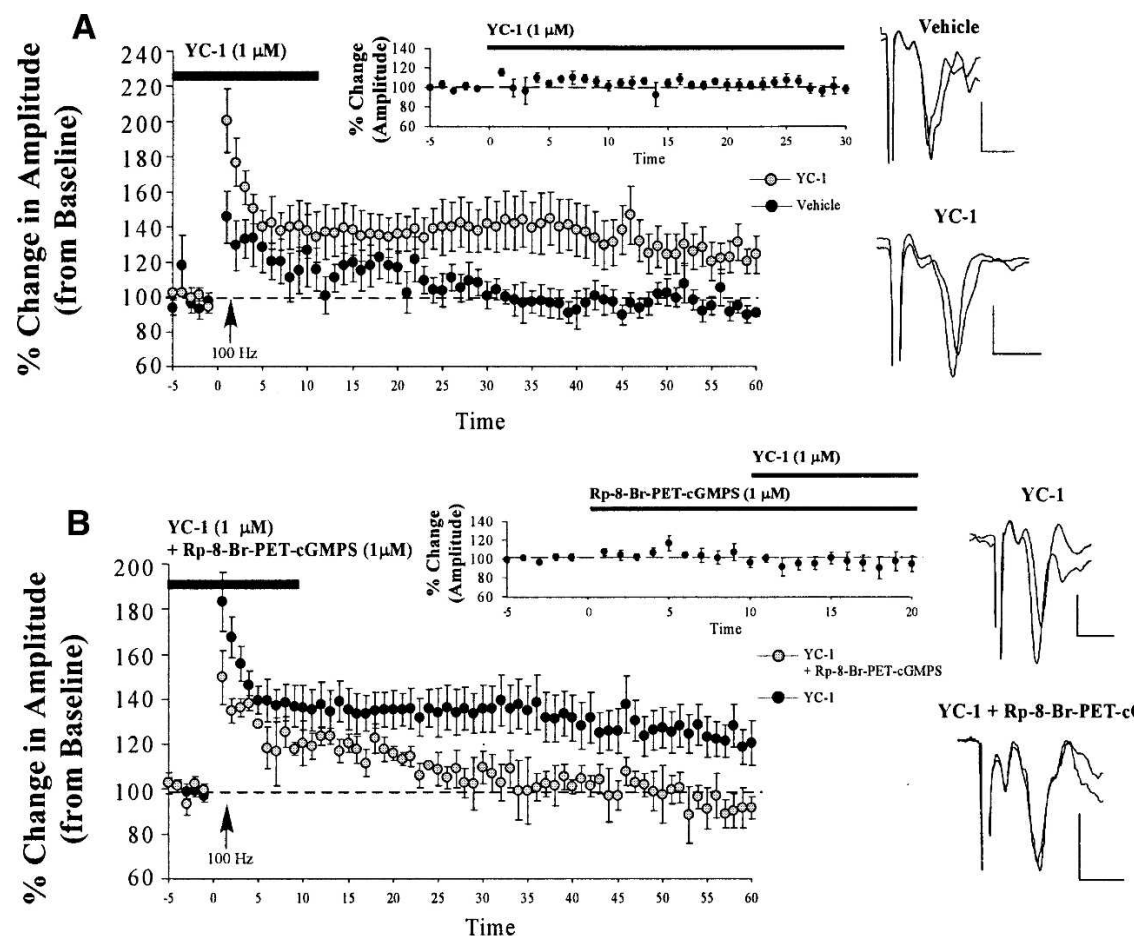

YC-1 + Rp-8-Br-PET-cGMPS
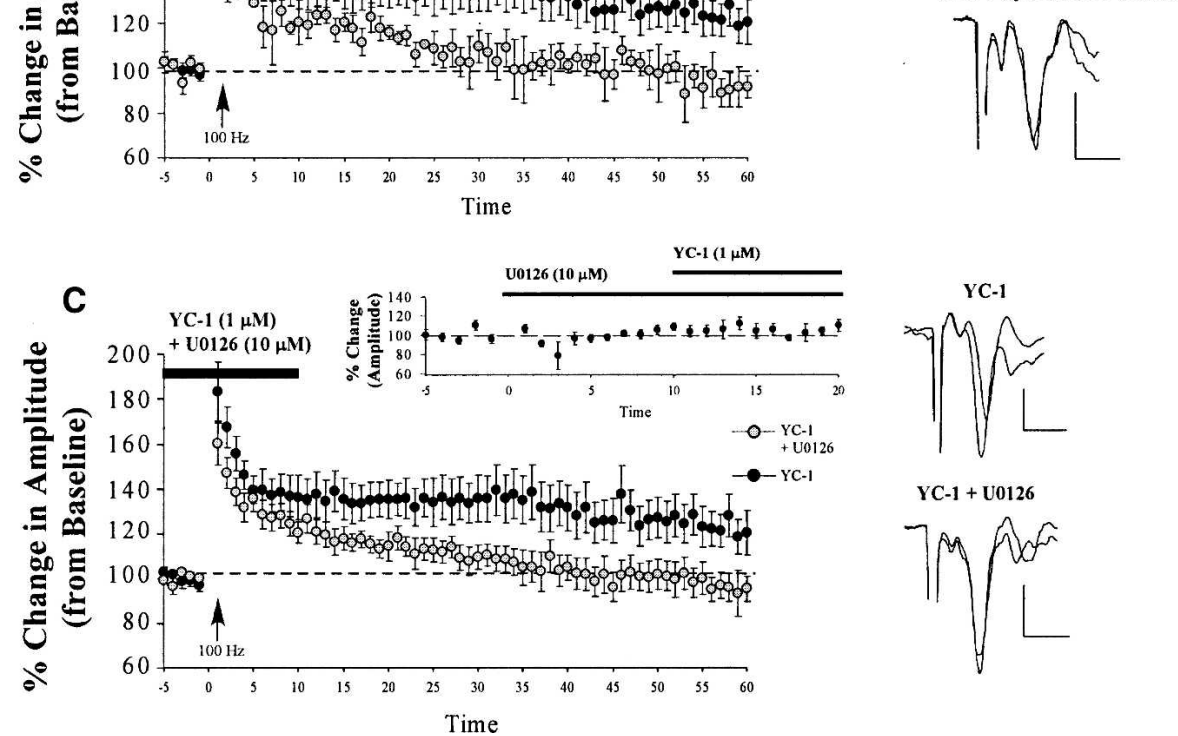

Figure 6. LTP at thalamic inputs is enhanced by activating the sGC-cGMP-PKG signaling pathway $(A)$ Mean $( \pm$ SEM) percentage field potential amplitude (relative to baseline) in slices treated with vehicle ( $n=6$; black circles) or $1 \mu \mathrm{M} \mathrm{YC-1}(n=8$; gray circles). Traces from an individual experiment before and 50 min following tetanic stimulation and transmission following 20 min of bath application of drug are shown in the inset. $(B)$ Mean $( \pm S E M)$ percentage field potential amplitude (relative to baseline) in slices treated with $1 \mu \mathrm{M}$ YC-1 $(n=13$; black circles) or $1 \mu \mathrm{M} \mathrm{YC-1}+1 \mu \mathrm{M}$ Rp-8-Br-PETcGMPS ( $n=5$; gray circles). Traces from an individual experiment before and 50 min following tetanic stimulation and transmission following $20 \mathrm{~min}$ of bath application of drug are shown in the inset. (C) Mean $( \pm$ SEM) percentage field potential amplitude (relative to baseline) in slices treated with $1 \mu \mathrm{M}$ YC-1 ( $n=13$; black circles) or $1 \mu \mathrm{M}$ YC-1 + $10 \mu \mathrm{M}$ U0126 $(n=9$; gray circles). Traces from an individual experiment before and 50 min following tetanic stimulation and transmission following 20 min of bath application of drug are shown in the inset. Note that the experiments in $B$ and $C$ were run simultaneously, so each experiment shares one control group (here graphed twice for the sake of clarity). Scale, $0.2 \mathrm{mV}$ by $10 \mathrm{msec}$.

\section{Slice electrophysiology}

We next examined the effects of PKG activation on synaptic plasticity at thalamic inputs to the LA. For this experiment, we used a low concentration of 8-Br-cGMP $(1 \mu \mathrm{M})$ that is highly specific to $P K G$, but has little affinity for other kinases such as PKA (Lu et al. 1999). Bath application of 8-Br-cGMP was found to enhance LTP at thalamic inputs to the LA compared with slices treated with ACSF only (Fig. 5I). As expected, our control (ACSF)-treated slices failed to exhibit potentiation using the weaker LTP induction protocol. Control slices showed $98.06 \pm 4.03 \%$ potentiation, which did not differ significantly from baseline $\left(t_{(5)}=0.23\right)$. In contrast, slices treated with 8-Br-cGMP exhibited enhanced LTP, potentiating to $114 \pm 6.33 \%$, which differed significantly from both baseline $\left(t_{(6)}=2.09, P<0.05\right.$; one-tailed) and the level of LTP in control slices $\left(t_{(11)}=1.99, P<0.05\right.$; one-tailed).

To further explore the role of PKG in amygdala LTP, we next examined the effect of YC-1, an sGC activator, on LTP at thalamo-LA synapses. Similar to the findings with the PKG activator, YC-1 relative to slices treated with ACSF (Fig. $6 \mathrm{~A})$. As before, the control slices failed to exhibit LTP $(96.47 \pm 5.04 \%)$, which did not differ significantly from baseline $\left(t_{(5)}=0.10\right)$. In contrast, the YC-1-treated slices potentiated to $124.83 \pm 3.84 \%$, which differed significantly from baseline $\left(t_{(7)}=2.62, P<0.05\right)$. The YC-1treated slices were also found to exhibit significantly higher levels of LTP than control slices $\left(t_{(12)}=2.42\right.$, $P<0.05)$. Thus, activation of both PKG and sGC enhanced LTP at thalamic inputs to the LA.

To verify whether the sGC activator was, in fact, enhancing LTP by activating PKG, we next examined the effects of bath application of a drug combination of YC-1 (sGC activator) and Rp-8-Br-PETcGMPS (PKG inhibitor) on LTP at thalamic inputs. The sGC activator YC-1, which was found to enhance LTP in slices (Fig. 6A), was perfused in conjuncwas found to enhance thalamo-LA LTP 
tion with the PKG inhibitor Rp-8-Br-PET-cGMPS, previously shown to inhibit LTP in slices (Fig. 3B), to determine whether the LTP enhancement induced by YC-1 could be blocked by simultaneously inhibiting PKG (Fig. 6B). Indeed, this hypothesis was supported. The slices perfused with YC-1 alone exhibited $124.10 \pm 8.46 \%$ potentiation, which differed significantly from baseline $\left(t_{(12)}=2.85, P<0.05\right)$. In contrast, the slices perfused concurrently with YC-1 and Rp-8-Br-PET-cGMPS exhibited $93.63 \pm 7.25 \%$ potentiation, which did not differ significantly from baseline $\left(t_{(4)}=0.70\right)$, but did differ significantly from the YC-1-treated group $\left(t_{(16)}=2.09, P<0.05\right.$; one-tailed $)$.

Importantly, none of the drugs used in these experiments, either alone or in combination, were found to have effects on routine transmission at thalamo-LA synapses. Bath application of 8-Br-cGMP or YC-1 alone (Figs. 5I, 6A, insets) failed to affect the baseline amplitude of evoked responses (8-Br-cGMP: $t_{(6)}=0.16$; YC-1: $t_{(6)}=0.42$ ). This pattern of findings indicates that it was the combination of weak tetanus and drug that promoted LTP at thalamo-LA synapses, not the perfusion of the drug alone. Further, co-application of both YC-1 and Rp-8-Br-PET-cGMPS also failed to affect baseline evoked responses $\left(t_{(4)}=0.58\right.$; Fig. $6 \mathrm{~B}$, inset), indicating that the reversal of YC-1 induced LTP in the LA was not due to impaired synaptic transmission as a result of perfusion of two different drugs onto the slice.

These findings, together with those of our experiments examining the effects of inhibition of sGC-cGMP-PKG signaling (above), suggest a crucial role for PKG and its upstream regulators in fear memory consolidation and LTP at thalamic inputs to the LA.

\section{The NO-cGMP-PKG signaling pathway promotes synaptic plasticity and fear memory consolidation via activation of ERK/MAPK}

Our experiments collectively suggest that signaling via NO-cGMPPKG regulates fear memory consolidation and associated synaptic plasticity in the LA. In this final series of experiments, we wanted to explore a potential mechanism by which LTP and fear memory consolidation might be promoted by NO-cGMP-PKG signaling in the LA. A recent study has suggested that ERK/MAPK is a critical downstream mediator of NO signaling in the hippocampus (Chien et al. 2003). In that study, YC-1-induced enhancement of LTP in hippocampal area CA1 was shown to be reversed by the MEK inhibitor PD098059. Further, LTP induced by coperfusion of YC-1 and the NO donor Na-nitroprusside led to significant activation of both ERK and the transcription factor CREB in CA1 neurons (Chien et al. 2003). These findings suggest that the NO-cGMP-PKG signaling pathway might promote synaptic plasticity and memory consolidation, in part, by activation of the MEK-ERK-CREB signaling cascade, a signaling cascade that has been widely implicated in memory consolidation, including fear memory consolidation (Atkins et al. 1998; Schafe et al. 2000; Josselyn et al. 2001). To evaluate this possibility in our system, we first used slice-recording methods to determine whether YC-1induced enhancement of LTP at thalamic inputs to the LA could be reversed by concurrent administration of the MEK inhibitor U0126 $(5 \mu \mathrm{M})$. Next, we examined whether ERK activation following fear conditioning is impaired, or enhanced, respectively, under the influence of the PKG inhibitor Rp-8-Br-PET-cGMPS (1 $\mu \mathrm{g})$ or the PKG activator 8-Br-cGMP $(10 \mu \mathrm{g})$.

\section{Slice electrophysiology}

In our slice electrophysiology experiment, the sGC activator YC-1 $(1 \mu \mathrm{M})$ was perfused over the slice in conjunction with the MEK inhibitor U0126 (5 $\mu \mathrm{M}$, a concentration that effectively blocks LTP in the LA) (Schafe et al. 2000). The findings revealed that adding U0126 to the bath effectively blocked the enhancement of LTP by YC-1 (Fig. 6C). The slices perfused with YC-1 alone resulted in a potentiation of $124.10 \pm 8.46 \%$, which differed significantly from baseline $\left(t_{(12)}=2.85, P<0.05\right)$. In contrast, the slices perfused concurrently with YC-1 and U0126 failed to potentiate $(97.86 \pm 7.0 \%$, which did not differ significantly from baseline, $\left.t_{(8)}=0.33\right)$. In addition, the two groups differed significantly from each other $\left(t_{(20)}=2.23, P<0.05\right)$. Importantly, co-perfusion of YC-1 and U0126 did not affect routine transmission at thalamo-LA inputs alone (Fig. 6C, inset). An analysis of the field-potential amplitude indicated that there was no significant difference before and after bath application of the $\operatorname{drug}\left(t_{(5)}=0.98\right)$.

These results suggest an interaction between the NO signaling pathway and the ERK/MAPK cascade in LTP at thalamo-LA synapses, suggesting that activation of the ERK/MAPK cascade is one mechanism by which the NO signaling pathway affects learning and memory in the LA.

\section{Fear conditioning and Western blotting}

The results of our slice electrophysiology experiment suggested the ERK/MAPK cascade as a possible mechanism by which the NO signaling pathway affects synaptic plasticity, and possibly memory formation, in the LA. As a more direct test of this hypothesis, we next examined ERK activation in fear-conditioned rats with or without infusion of either the PKG inhibitor Rp-8Br-PET-cGMPS or the PKG activator 8-Br-cGMP (Fig. 7A,D). Rats were given intra-LA infusion of either vehicle (ACSF), the PKG inhibitor Rp-8-Br-PET-cGMPS ( $1 \mu \mathrm{g}$, the dose that we determined to impair fear memory consolidation) (Fig. 1), or the PKG activator 8-Br-cGMP $(10 \mu \mathrm{g}$, the dose that we determined to enhance fear memory consolidation) (Fig. 5) 60 min prior to fear conditioning. Rats were then trained with three or two tone-shock pairings as in our behavioral experiments (above, Figs. 1A, 5A) and sacrificed at $1 \mathrm{~h}$ after training, a time point that we have previously shown is optimal for observing training-induced increases in ERK activation in the LA (Schafe et al. 2000). Western blotting on LA tissue taken from around the cannula tips was performed to determine whether training-induced activation of ERK/MAPK is impaired following infusion of the PKG inhibitor or further enhanced when animals are trained under the influence of the PKG activator.

The findings of the Western blot PKG inhibitor experiment are depicted in Figure 7B. Relative to vehicle-infused controls, rats infused with Rp-8-Br-PET-cGMPS prior to training exhibited significant decreases in both phospho-ERK1 and phospho-ERK2 immunoreactivity in the LA. The ANOVA revealed a significant effect for drug (vehicle vs. Rp-8-Br-PET-cGMPS; $F_{(1,41)}=4.92$, $P<0.05$ ), a nonsignificant effect for kinase (ERK1 vs. ERK2; $\left.F_{(1,41)}=0.56, P>0.05\right)$, and a nonsignificant drug by kinase interaction $\left(F_{(1,41)}=0.56, P>0.05\right)$. Furthermore, this impairment of ERK activation cannot be accounted for by differences in total ERK/MAPK. Total ERK/MAPK levels, expressed relative to the loading control GAPDH, were not significantly changed from vehicle levels following infusion of Rp-8-Br-PET-cGMPS (Fig. 7C). The ANOVA revealed a nonsignificant effect for drug $\left(F_{(1,41)}=2.62, P>0.05\right)$, a nonsignificant effect for kinase $\left(F_{(1,41)}=0.009, P>0.05\right)$, and a nonsignificant drug by kinase interaction $\left(F_{(1,41)}=0.009, P>0.05\right)$.

The findings of the Western blot PKG activator experiment showed the reverse of the PKG inhibitor experiment (Fig. 7E). Relative to vehicle-infused controls, rats infused with 8-Br-cGMP prior to training exhibited significant increases in both phosphoERK1 and phospho-ERK2 immunoreactivity in the LA. The ANOVA revealed a significant effect for drug (vehicle vs. 8-BrcGMPS; $\left.F_{(1,36)}=5.70, P<0.05\right)$, a nonsignificant effect for kinase 


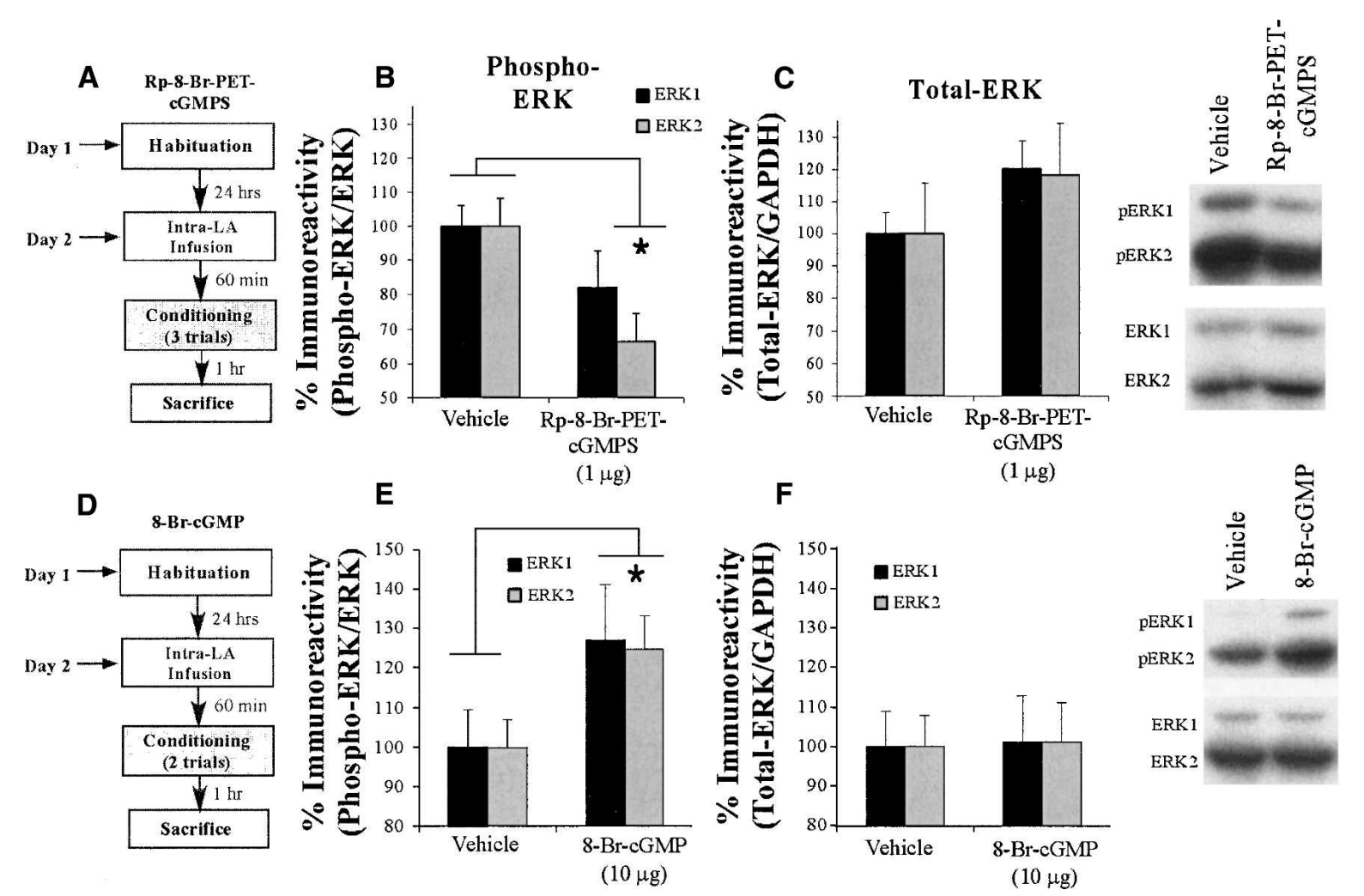

Figure 7. Intra-LA infusion of a PKG inhibitor or activator impairs or enhances, respectively, ERK phosphorylation in the LA following fear conditioning. (A) Schematic of behavioral protocol for PKG inhibitor. Rats were given intra-LA infusion of the vehicle or $1 \mu \mathrm{g} / \mathrm{side}$ Rp-8-Br-PET-cGMPS then trained using a three-trial conditioning protocol and sacrificed $1 \mathrm{~h}$ later. $(B)$ Mean $( \pm$ SEM) percent phospho-ERK immunoreactivity from LA punches taken from rats given intra-LA infusions of ACSF (vehicle; $n=13$ ) or $1 \mu \mathrm{g} /$ side Rp-8-Br-PET-cGMPS $(n=10)$. Here, phospho-ERK levels have been normalized to total ERK levels for each sample. (C) Mean $( \pm$ SEM) percent total-ERK immunoreactivity from LA punches taken from rats given intra-LA infusions of ACSF (vehicle; $n=13$ ) or $1 \mu \mathrm{g} /$ side Rp-8-Br-PET-cGMPS $(n=10)$. Here, total ERK levels have been normalized to GAPDH levels for each sample. Representative blots for both phospho-ERK (pERK) and ERK can be viewed at the right. $(D)$ Schematic of behavioral protocol for PKG activator. Rats were given intra-LA infusion of the vehicle or $10 \mu \mathrm{g} /$ side 8-Br-cGMP then trained using a two-trial conditioning protocol and sacrificed $1 \mathrm{~h}$ later. $(E)$ Mean $( \pm$ SEM) percent phospho-ERK/ERK immunoreactivity from LA punches taken from rats given intra-LA infusions of ACSF (vehicle; $n=11)$ or $10 \mu \mathrm{g} / \mathrm{side} 8$-Br-cGMP $(n=9)$. $(F)$ Mean ( \pm SEM) percent total-ERK/GAPDH immunoreactivity from LA punches taken from rats given intra-LA infusions of ACSF (vehicle; $n=11$ ) or 10 $\mu \mathrm{g} /$ side 8-Br-cGMP $(n=9)$. Representative blots for both phospho-ERK (pERK) and ERK can be viewed at the right. $\left(^{*}\right) P<0.05$ relative to vehicle-infused rats.

(ERK1 vs. ERK2; $\left.F_{(1,36)}=0.014, P>0.05\right)$, and a nonsignificant drug by kinase interaction $\left(F_{(1,36)}=0.014, P>0.05\right)$. Again, this increase is not accounted for by differences in total ERK. Total ERK/MAPK levels were not significantly changed from vehicle levels following infusion of 8-Br-cGMP (Fig. 7F). The ANOVA revealed a nonsignificant effect for drug $\left(F_{(1,36)}=0.011\right.$, $P>0.05)$, a nonsignificant effect for kinase $\left(F_{(1,36)}=0.006\right.$, $P>0.05)$, and a nonsignificant drug by kinase interaction $\left(F_{(1,36)}=0.006, P>0.05\right)$.

Together with our LTP experiment, these results suggest that the NO-cGMP-PKG signaling pathway affects fear memory consolidation and synaptic plasticity in the LA, in part, by promoting the activation of ERK/MAPK.

\section{Discussion}

We have recently shown that NO signaling in the LA is critical for synaptic plasticity and fear memory consolidation (Schafe et al. 2005a). The current study sought to further examine the NO signaling pathway in fear memory formation by examining downstream targets of $\mathrm{NO}$ signaling in the LA, including the role of PKG and its immediate upstream regulators. Collectively, our findings suggest that signaling via PKG regulates both fear memory consolidation and synaptic plasticity in the LA, provid- ing further evidence that the NO-cGMP-PKG signaling pathway is critical for fear memory formation.

\section{The NO-cGMP-PKG signaling pathway is required for fear memory formation}

In our behavioral experiments, we examined the role of PKG signaling specifically in auditory fear conditioning and in the LA. We found that intra-LA infusion of the PKG inhibitor Rp-8-BrPET-cGMPS impaired fear memory formation. Specifically, LTM was impaired, while STM, measured within hours of training, was intact. In contrast, infusion of the PKG activator 8-Br-cGMP enhanced LTM, but similarly had no effect on STM. The fact that fear acquisition and STM formation was left intact after manipulation of PKG signaling rules out possible nonspecific explanations of the drug effect, including the possibility that either drug influenced fear acquisition by impairing (or enhancing) sensory and/or performance factors, including the ability of the LA to process tone and shock information.

Intact acquisition and STM formation also rules out potential effects of manipulation of the NO signaling pathway in the LA on motivational factors that might influence fear conditioning. A previous study has shown that intra-LA infusion of a NOS inhibitor produces an anxiogenic state in rats as measured by the 
elevated plus maze (Monzón et al. 2001). Such an effect might therefore also be predicted following infusion of an inhibitor of PKG. Given the fact that anxiogenic drugs, including dopamine receptor agonists (Sullivan et al. 2003), adrenergic agonists (Davis et al. 1979), and acutely administered SSRIs (Burghardt et al. 2004), are all known to facilitate, rather than impair fear conditioning, our results are not consistent with this finding. Further, the Monzón et al. (2001) findings would suggest that activation of PKG in the LA should be anxiolytic, a conclusion that our data are also clearly not consistent with. Rather, our findings, when considered together with our recent findings that showed impaired fear memory consolidation following infusion of an inhibitor of NOS or of a membrane-impermeable scavenger of NO (Schafe et al. 2005a), provide strong evidence that NO signaling is required for fear memory consolidation and associated synaptic plasticity in the amygdala.

\section{The NO-cGMP-PKG signaling pathway is required for synaptic plasticity in the LA, but only at thalamic inputs}

In our neurophysiology experiments, we found that pharmacological blockade or activation of the cGMP-PKG signaling pathway impaired or enhanced LTP at thalamic inputs to the LA, respectively. These findings are consistent with those of our recent findings, which showed that LTP occludes paired pulse facilitation at thalamic inputs to the LA, and that bath application of an inhibitor of NOS or a scavenger of NO impairs thalamo-LA LTP (Schafe et al. 2005a). Further, the fact that LTP was impaired whether Rp-8-Br-PET-cGMPS was present in the bath or in the recording pipette suggests that the NO-cGMP-PKG signaling pathway acts, at least in part, at postsynaptic sites to promote synaptic plasticity in the LA. Given that Rp-8-Br-PET-cGMPS is membrane permeable, however, it is difficult to conclude with certainty that post-synaptic injection of Rp-8-Br-PET-cGMPS impairs LTP by acting in the postsynaptic cell.

Of particular interest is our finding that the NO-cGMP-PKG signaling pathway selectively impairs LTP at thalamic inputs to the LA; LTP at cortical inputs is not affected, in spite of the fact that both thalamic and cortical inputs are known to synapse onto the same cells in the LA (Li et al. 1996). These findings are consistent with that of an earlier study that also failed to find effects of NO manipulation on cortical-LA LTP (Watanabe et al. 1995), and suggests a fairly specific role for NO signaling at thalamo-LA synapses. This type of pathway specificity has also been observed in the hippocampus, where LTP at apical, but not basilar synapses on CA1 pyramidal cells requires NO signaling (O'Dell et al. 1994; Son et al. 1996). This finding is somewhat surprising, however, in light of studies that have shown that LTP at cortical inputs to the LA is likely subserved by a presynaptic mechanism. For example, Huang and Kandel (1998) showed that LTP occludes paired-pulse facilitation at cortical inputs to the LA. Further, bath application, but not postsynaptic injection of a PKA inhibitor impairs LTP at cortico-LA inputs (Huang and Kandel 1998). Conversely, bath application of forskolin, a PKA activator, in the presence of antagonists of postsynaptic NMDAR and AMPAR receptors, induces LTP and occludes PPF at cortical inputs (Huang and Kandel 1998), suggesting that the presynaptic component of LTP in this pathway is PKA dependent. More recently, Tsvetkov et al. (2002) showed that auditory fear conditioning itself, in addition to LTP, occludes paired-pulse facilitation at cortical inputs to LA (Tsvetkov et al. 2002), a finding consistent with that of McKernan and Shinnick-Gallagher (1997). These findings suggest that while synaptic plasticity at cortical inputs to the LA may involve a presynaptic component,
NO signaling is not a mechanism by which this presynaptic component is acquired or expressed.

As in previous studies that have directly examined the relationship between LTP and memory formation in the amygdala, LTP in our slices treated with either antagonists or agonists of the cGMP-PKG signaling pathway decayed (or was enhanced) at a much faster time course than fear memory after the same manipulations. This is not a novel finding, but is, in fact, frequently observed in studies that have directly compared the time course of memory and LTP decay under the influence of the same manipulation (Schafe et al. 2001). Many compounds that impair long-term but not short-term memory have been shown to block LTP within minutes after induction. In the in vitro amygdala preparation, for example, bath application of antagonists to Ltype voltage-gated calcium channels or inhibitors of protein synthesis, PKA, ERK/MAPK activity or NO signaling begins to impair LTP immediately after induction (Huang et al. 2000; Schafe et al. 2000, 2005a; Bauer et al. 2002), whereas intra-amygdala infusion of the same compounds in behavioral experiments produces memory deficits that emerge between 6 and $24 \mathrm{~h}$ after conditioning (Schafe and LeDoux 2000; Schafe et al. 2000, 2005a; Bauer et al. 2002). Any number of factors might account for the temporal discrepancy between LTP and memory formation. For example, in vitro LTP studies use artificial patterns of electrical stimulation to induce LTP, which may be very different from natural activity patterns that occur in the LA of behaving animals during CS-US pairing. Further, neurons undergo significant trauma during preparation of brain slices for in vitro experiments, and they are disconnected from many of the modulatory inputs that are normally present in vivo. These factors may be responsible for quantitative differences in the time course of the effects of drugs on protein synthesis-dependent LTP and LTM formation, even though both phenomena involve qualitatively similar molecular signaling pathways. It is clear that a complete understanding of how synaptic plasticity in the LA and fear conditioning are related will require attention to the relationship between naturally occurring plasticity in the LA and fear memory formation (Schafe et al. 2005b).

\section{The NO-cGMP-PKG signaling pathway acts via the ERK/MAPK signaling cascade}

The findings of the present study are the first to point to a potential mechanism by which the NO signaling pathway affects fear memory consolidation and synaptic plasticity in the LA. Coapplication of the MEK inhibitor U0126 reversed the LTPenhancing effects of the SGC inhibitor YC-1 in LA slices, indicating that YC-1 enhancement of synaptic plasticity in the LA is dependent on ERK activation. In behavioral experiments, intraLA infusion of the PKG activator 8-Br-cGMP enhanced traininginduced ERK activation in LA homogenates, while infusion of the PKG inhibitor Rp-8-Br-PET-cGMPS resulted in impaired traininginduced ERK activation in the LA. Together, these findings suggest that the NO-cGMP-PKG signaling pathway acts to promote fear memory formation and synaptic plasticity in the LA, in part by activation of ERK in LA neurons. This pattern of findings is in agreement with previous studies that have suggested that one of the mechanisms by which NO exerts its effects on synaptic plasticity and memory is by engaging parallel activation of "classical" signaling cascades, including ERK/MAPK and the transcription factor CREB (Lu et al. 1999; Chien et al. 2003). It has long been known that ERK is a critical signaling cascade in fear conditioning (Atkins et al. 1998; Schafe et al. 2000), but the critical upstream mediators of ERK signaling in the LA have not been identified. The findings of the present study provide some of the first support that $\mathrm{NO}$ and its downstream effectors may be one of 
these critical mediators, although additional experiments will be required to explore how PKG and ERK might interact in the signaling cascade. While the mechanism by which NO signaling leads to ERK activation in the LA during fear learning is presently unknown, previous studies have suggested that PKG or its downstream substrates can activate Raf-1, an upstream regulator of ERK1/2 (Hood and Granger 1998), or inhibit protein phosphatase-1 (Hall et al. 1998), which may indirectly regulate ERK1/2.

Importantly, while the findings of the present study clearly suggest that activation of ERK-mediated signaling in LA neurons is one mechanism by which the NO-cGMP-PKG signaling pathway promotes fear memory consolidation, they do not rule out the possibility that NO signaling in the LA also plays a role in presynaptic aspects of plasticity in the LA. Our Western blotting experiments (Fig. 7), for example, indicate that PKG signaling regulates ERK activation in LA homogenates, but the possibility remains that all or part of this regulation might be localized to presynaptic terminals in the LA. Further, while previous studies from our laboratory have clearly implicated the importance of ERK activation in the LA in fear memory consolidation (Schafe et al. 2000), more recent findings have also implicated the importance of ERK-mediated signaling in the auditory thalamus (Apergis-Schoute et al. 2005). In those experiments, intra-thalamic infusion of the MEK inhibitor U0126 or the mRNA synthesis inhibitor DRB impaired fear memory consolidation, while infusion of the protein synthesis inhibitor anisomycin did not (Apergis-Schoute et al. 2005). These findings suggest that while ERKdriven transcription in auditory thalamic neurons is critical for fear memory consolidation, it is not itself a site of fear memory storage. Interestingly, intra-thalamic infusion of U0126 was also observed to impair LTP at thalamic inputs to the LA, suggesting that the role of ERK-dependent signaling in the auditory thalamus is to promote presynaptic aspects of plasticity at thalamo-LA synapses (Apergis-Schoute et al. 2005). At present, it remains unclear how ERK-driven transcription in the thalamus is engaged by fear conditioning. However, it may well be possible that NO signaling in the LA engages, in parallel, ERK activation in both LA and the auditory thalamus during the process of fear memory consolidation. In this manner, NO may serve as the link between intracellular signaling in the LA and that in its afferent targets during the process of fear memory consolidation. Further experiments will be necessary to examine this possibility.

In summary, the results of the present study clearly suggest that signaling via PKG regulates long-term potentiation and fear memory consolidation in the LA. These findings provide further evidence for a role for the NO-cGMP-PKG signaling pathway in fear memory formation and associated synaptic plasticity in the LA and make an additional contribution toward understanding the cellular and molecular processes underlying emotional memory formation in the amygdala.

\section{Materials and Methods}

\section{Subjects}

Adult male Sprague-Dawley rats (Harlan), weighing 300-325 g, were housed individually in plastic cages and maintained on a 12:12 h light/dark cycle. Food and water were provided ad libitum throughout the experiment.

\section{Drugs}

In the behavioral experiments, we used the PKG inhibitor guanosine $3^{\prime}, 5^{\prime}$-cyclic monophosphorothioate, $\beta$-Phenyl-1, $\mathrm{N}^{2}$ etheno-8-bromo-, Rp-Isomer, sodium salt (Rp-8-Br-PET-cGMPS; Calbiochem, Cat. No. 370679) and the PKG activator guanosine 3',5'-cyclic monophosphate, 8-Bromo-, sodium salt (8-Br-cGMP; Calbiochem, Cat. no. 203820). The drugs were dissolved in dis- tilled water in a stock concentration of either $2 \mu \mathrm{g} / \mu \mathrm{L}(\mathrm{Rp}-8-\mathrm{Br}-$ PET-cGMPS) or $20 \mu \mathrm{g} / \mu \mathrm{L}$ (8-Br-cGMP).

In the electrophysiological experiments, we used the PKG drugs outlined above in addition to the sGC activator 3-(5'Hydroxymethyl-2'-furyl)-1-benzylindazole (YC-1; Calbiochem Cat. No. 688100), the MEK inhibitor U0126 (Promega, Cat. No. V1121), the NOS inhibitor 7-nitroindazole (7-Ni; Calbiochem Cat. No. 483400), the sGC inhibitor 6-Anilino-5,8-quinolinequinone (LY 83583; Cat. No. 440205), and the NMDA receptor antagonist D-(-)-2-Amino-5-phosphonopentanoic Acid (AP-5; Calbiochem Cat. No.165304). All drugs, with the exception of Rp-8-Br-PETcGMPS, were diluted in 100\% DMSO into stock concentrations of $1 \mathrm{mM}$ (8-Br-cGMP and YC-1), $5 \mathrm{mM}$ (LY 83583), $10 \mathrm{mM}$ (U0126), $30 \mathrm{mM}$ (7-Ni), or $40 \mathrm{mM}$ (AP-5). The Rp-8-Br-PET-cGMPS, because it is water soluble, was diluted in distilled water in a stock concentration of $1 \mathrm{mM}$. For use in experiments, all drugs were further diluted in ACSF for perfusion over the slice at a final concentration of 1000-fold less than the stock concentration.

\section{Surgical procedures}

Under a mixture of Ketamine $(100 \mathrm{mg} / \mathrm{kg})$ and Xylazine $(6.0$ $\mathrm{mg} / \mathrm{kg}$ ) anesthesia, rats were implanted bilaterally with 23-gauge stainless-steel guide cannulas aimed at the LA. The coordinates for the LA were: $-3.2 \mathrm{~mm}, \pm 5.0 \mathrm{~mm},-8.0 \mathrm{~mm}$ relative to Bregma. The guide cannulas were fixed to screws in the skull using a mixture of acrylic and dental cement, and a 28-gauge dummy cannula was inserted into each guide cannula to prevent clogging. Rats were given Buprenex $(0.2 \mathrm{mg} / \mathrm{kg})$ as an analgesic and given at least $5 \mathrm{~d}$ to recover prior to experimental procedures. All procedures were conducted in accordance with the National Institutes of Health "Guide for the Care and Use of Experimental Animals" and were approved by the Yale University Animal Care and Use Committee.

\section{Behavioral procedures}

On the day prior to conditioning, rats were habituated to the conditioning chamber and to dummy cannula removal for a minimum of $10 \mathrm{~min}$. The following day, rats were given an intraLA infusion of either $0.5 \mu \mathrm{L} 0.9 \% \mathrm{NaCl}$ (vehicle), Rp-8-Br-PETcGMPS $(0.1$ or $1.0 \mu \mathrm{g} /$ side in $0.5 \mu \mathrm{L}$ ), or $8-\mathrm{Br}$-cGMP $(1.0$ or 10 $\mu \mathrm{g} /$ side in $0.5 \mu \mathrm{L}$ ). All infusions were given at a rate of 0.25 $\mu \mathrm{L} / \mathrm{min}$. Injectors remained in the cannulas for $1 \mathrm{~min}$ after drug infusion to allow diffusion of the drug from the tip. Sixty minutes following drug infusion, rats infused with Rp-8-Br-PETcGMPS (and their respective Vehicle controls) were trained with three conditioning trials consisting of a $20-\mathrm{sec}, 5-\mathrm{kHz}, 75-\mathrm{dB}$ tone that co-terminated with a 1.0 -sec, $0.5-\mathrm{mA}$ foot shock (ITI $=120$ sec). Rats infused with 8-Br-cGMP (and their respective controls) were trained as above, but with only two training trials to maximize our ability to observe enhancements in fear acquisition and/or retention.

Testing for conditioned fear to the tone occurred at $1,3,6$, and $24 \mathrm{~h}$ following training. For each test, rats were placed in a distinctive environment that was dark and consisted of a flat black plastic floor that had been washed with a peppermintscented soap. For the 1-, 3-, and 6-h retention tests, rats were exposed to three conditioned stimulus (CS) tones $(5 \mathrm{kHz}, 75 \mathrm{~dB}$, $20 \mathrm{sec}$ ). For the $24 \mathrm{~h}$ test, rats were exposed to $10 \mathrm{CS}$ tones for the Rp-8-Br-PET-cGMPS experiment and to three tones for the 8-BrcGMP experiment. For each tone test, we measured the rats' freezing behavior, defined as a lack of all movement, with the exception of that required for respiration, and expressed this measure as a percentage of the total CS presentation time. Freezing was calculated from activity counts measured automatically during each CS presentation by Coulbourne Instruments Activity Monitors (Model \# H10-24A) mounted at the top of each of the behavioral chambers. For each memory test, freezing scores for each trial were averaged together as a single score for each rat. All data were analyzed with Analysis of Variance (ANOVA) and Duncan's post-hoc $t$-tests. For behavioral experiments involving multiple trial comparisons (Figs. 1, 2, 4), repeated measures ANOVA was used. Differences were considered significant if $P<0.05$. 
At the end of the behavioral experiment, rats were sacrificed by an overdose of chloral hydrate $(600 \mathrm{mg} / \mathrm{kg})$ and perfused with $0.9 \%$ saline followed by $10 \%$ buffered formalin. Nissl staining and light microscopy were used to verify the location of the cannula tips within the amygdala.

\section{Slice electrophysiology}

Male Sprague-Dawley rats (3-5 wk old) were deeply anesthetized with ketamine, and the brain was rapidly removed and transferred to ice-cold ACSF containing (in millimolars): $115 \mathrm{NaCl}, 3.3$ $\mathrm{KCl}, 1 \mathrm{MgSO}_{4}, 2 \mathrm{CaCl}_{2}, 25.5 \mathrm{NaHCO}_{3}, 1.2 \mathrm{NaH}_{2} \mathrm{PO}_{4}$, and 10 glucose, equilibrated with $95 \% \mathrm{O}_{2} / 5 \% \mathrm{CO}_{2}$. Coronal slices $(400-\mu \mathrm{M}$ thick) containing the amygdala were cut and recovered in a holding chamber at $32^{\circ} \mathrm{C}-34^{\circ} \mathrm{C}$ for $30 \mathrm{~min}$ and were then allowed to return to room temperature for at least another $30 \mathrm{~min}$ before recording. An upright microscope equipped with infrared differential interference contrast optics (IR-DIC) was used to perform whole-cell patch or field recordings under visual guidance. For field recording experiments, glass-recording electrodes were filled with ACSF. For whole-cell experiments, electrodes were filled with (in millimolars): $130 \mathrm{~K}$-Gluconate, $0.6 \mathrm{EGTA}, 2 \mathrm{MgCl}_{2}, 5$ $\mathrm{KCl}, 10$ HEPES, $2 \mathrm{Mg}$-ATP, $0.3 \mathrm{Na}_{3}$-GTP (pH 7.3, 290-300 mOsm). The electrodes typically had resistances of $4-8 \mathrm{M} \Omega$. For some experiments, we dissolved the PKG inhibitor Rp-8-Br-PET-cGMPS $(250 \mu \mathrm{M})$ in the internal solution. $\mathrm{pH}(7.3)$ and osmolarity (290$300 \mathrm{mOsm})$ were rechecked prior to using the drug in slicerecording experiments.

Stimuli (150- $\mu$ sec duration) were delivered through bipolar stainless steel electrodes. For "thalamic" recording experiments, stimulation electrodes were placed in the ventral striatum, just medial to the LA. This stimulating protocol activates fibers that originate, at least in part, in the auditory thalamus (Weisskopf et al. 1999). For "cortical" recording experiments, stimulation electrodes were placed in the external capsule, just dorsal to the LA. This stimulating protocol activates fibers that originate, at least in part, in the auditory cortex (Chapman et al. 1990). The stimulation was kept at a minimum and adjusted for each cell or slice to produce a reliable EPSP or field-evoked response that was $\sim 50 \%$ of the maximal response. Baseline responses were monitored at $0.06 \mathrm{~Hz}$. Following stabilization of baseline responses, one of three different LTP-induction protocols was used. For whole-cell recording experiments, LTP was induced by delivering trains of stimuli at $30 \mathrm{~Hz}$ given twice at a 20 -sec interval. This is a type of LTP induction protocol that, in the LA, is known to be NMDAR dependent (Bauer et al. 2002). For field recording experiments, we used a $100-\mathrm{Hz}$ tetanus given three times at $1-\mathrm{min}$ intervals at test intensity (weak protocol), or a $100-\mathrm{Hz}$ tetanus given three times at 1-min intervals at 50\% higher stimulation intensity (strong protocol). In our hands, this latter $100 \mathrm{~Hz}$ protocol produces a reliable LTP that lasts for at least $1 \mathrm{~h}$, whereas the weaker $100 \mathrm{~Hz}$ protocol declines to baseline within $\sim 30 \mathrm{~min}$. Recordings were made at baseline test intensity for an additional hour after LTP induction in field-recording experiments and for an additional $30 \mathrm{~min}$ in whole-cell experiments.

Picrotoxin $(50 \mu \mathrm{M})$ was included in the bath in all experiments to block fast GABAergic transmission. Each slice was recorded from only once, and thus, control and drug conditions were always from different slices. Typically, both vehicle and drug conditions were run from separate slices from the same animal on the same day. In control experiments, slices were perfused with ACSF $/ 50 \mu \mathrm{M}$ picrotoxin vehicle alone.

Data were acquired using Slice software (http://www. cns.nyu.edu/ sanes/slice_software/) written for Igor (NIH DC00540). EPSPs and field potentials were extracted from Igor and analyzed using Spike 2 software. In all experiments, the initial slope of the EPSP or amplitude of the negative-going field potential was measured, and LTP for each time point was expressed as a percentage of the pre-induction baseline. For analysis, measurements of evoked responses during the last $10 \mathrm{~min}$ of the recording session were compared with the last $5 \mathrm{~min}$ of the baseline period using Student's $t$-tests.

\section{Western blotting}

In Western blotting experiments, rats were cannulated as described above. Rats were habituated to handling, conditioning chambers, and dummy cannula removal for $2 \mathrm{~d}$ prior to training. On the training day, animals were given an intra-LA infusion of either ACSF (vehicle, prepared as described under slice electrophysiology procedures), the PKG inhibitor Rp-8-Br-PET-cGMPS ( $1 \mu \mathrm{g} / \mathrm{side}$ in $0.5 \mu \mathrm{L} ; 0.25 \mu \mathrm{L} / \mathrm{min})$, or the PKG activator 8 -BrcGMP $(10 \mu \mathrm{g} /$ side in $0.5 \mu \mathrm{L} ; 0.25 \mu \mathrm{L} / \mathrm{min})$. Injectors remained in the cannulas for $1 \mathrm{~min}$ after drug infusion to allow diffusion of the drug from the tip. Sixty minutes following drug infusion, rats were trained with three or two conditioning trials, respectively, consisting of a $20-\mathrm{sec}, 5-\mathrm{kHz}, 75-\mathrm{dB}$ tone that co-terminated with a $1.0-\mathrm{sec}, 0.5-\mathrm{mA}$ foot shock $($ ITI $=120 \mathrm{sec}$ ). One hour following training, animals were sacrificed using an overdose of chloral hydrate $(600 \mathrm{mg} / \mathrm{kg})$ and decapitated. Brains were frozen and stored at $-80^{\circ} \mathrm{C}$ until processed. Punches containing the LA were obtained with a 1-mm punch tool (Fine Science Tools) from 400$\mu \mathrm{m}$-thick sections taken on a sliding freezing microtome. Punches were manually dounced in $50 \mu \mathrm{L}$ of ice-cold hypotonic lysis buffer (10 mM Tris-Hcl, pH 7.5, 1 mM EDTA, $2.5 \mathrm{mM}$ sodium pyrophosphate, $1 \mathrm{mM}$ phenylmethylsulfonyl fluoride, 1 $\mathrm{mM} \beta$-glycero-phosphate, $1 \%$ Igepal CA-630, 1\% protease inhibitor cocktail [Sigma], and $1 \mathrm{mM}$ sodium orthovanadate). Protein concentrations were assessed and normalized across homogenates using a Bradford assay. Sample buffer was immediately added to the homogenates, and the samples were boiled for 4 min. Homogenates were electrophoresed on $10 \%$ Tris- $\mathrm{HCl}$ gels and blotted to Immobilon-P (Millipore). Western blots were blocked in 5\% milk and then incubated with an anti-phosphoMAPK (1:1000; Cell Signaling) or an anti-total MAPK antibody (1:1000; Cell Signaling). Blots were then incubated with antirabbit conjugated to horseradish peroxidase and developed using enhanced chemiluminescence (Pierce). Optical densities of the bands were analyzed using NIH Image software. To assess for changes in the activation of ERK/MAPK, phosphorylated kinase levels were normalized to total ERK levels. Activated ERK levels in drug-infused rats were then expressed as a percentage of those in vehicle-infused rats. To confirm that total ERK levels remained constant across infusions, blots were blocked in 5\% BSA in TTBS and reincubated in GAPDH antibody (1:5000; Abcam). Following incubation with anti-mouse conjugated to horseradish peroxidase, blots were developed identically to those processed for phospho-ERK and total ERK. Total ERK levels were then normalized to GAPDH levels for analysis.

\section{Acknowledgments}

This research was supported by the National Science Foundation (NSF 0444632 to G.E.S.), the National Institutes of Health (MH 073949 to G.E.S.), and by Yale University. K.T.O. is supported by a National Science Foundation Graduate Research Fellowship. We thank Grace Young and Kunmi Sobowale for technical assistance.

\section{References}

Apergis-Schoute, A.M., Debiec, J., Doyere, V., LeDoux, J.E., and Schafe, G.E. 2005. Auditory fear conditioning and long-term potentiation in the lateral amygdala require ERK/MAP kinase signaling in the auditory thalamus: A role for presynaptic plasticity in the fear system. J. Neurosci. 25: 5730-5739.

Arancio, O., Kiebler, M., Lee, C.J., Lev-Ram, V., Tsien, R.Y., Kandel, E.R., and Hawkins, R.D. 1996. Nitric oxide acts directly in the presynaptic neuron to produce long-term potentiation in cultured hippocampal neurons. Cell 87: 1025-1035.

Arancio, O., Antonova, I., Gambaryan, S., Lohmann, S.M., Wood, J.S., Lawrence, D.S., and Hawkins, R.D. 2001. Presynaptic role of cGMPdependent protein kinase during long-lasting potentiation. I. Neurosci. 21: 143-149.

Atkins, C.M., Selcher, J.C., Petraitis, J.J., Trzaskos, J.M., and Sweatt, J.D. 1998. The MAPK cascade is required for mammalian associative learning. Nat. Neurosci. 1: 602-609.

Bauer, E.P., Schafe, G.E., and LeDoux, J.E. 2002. NMDA receptors and L-type voltage-gated calcium channels contribute to long-term 
potentiation and different components of fear memory formation in the lateral amygdala. J. Neurosci. 22: 5239-5249.

Bernabeu, R., de Stein, M.L., Fin, C., Izquierdo, I., and Medina, J.H. 1995. Role of hippocampal NO in the acquisition and consolidation of inhibitory avoidance learning. Neuroreport 6: 1498-1500.

Bernabeu, R., Schmitz, P., Faillace, M.P., Izquierdo, I., and Medina, J.H. 1996. Hippocampal cGMP and cAMP are differentially involved in memory processing of inhibitory avoidance learning. Neuroreport 7: 585-588.

Bernabeu, R., Schroder, N., Quevedo, J., Cammarota, M., Izquierdo, I, and Medina, J.H. 1997. Further evidence for the involvement of a hippocampal cGMP / cGMP-dependent protein kinase cascade in memory consolidation. Neuroreport 8: 2221-2224.

Bohme, G.A., Bon, C., Lemaire, M., Reibaud, M., Piot, O., Stutzmann J.M., Doble, A., and Blanchard, J.C. 1993. Altered synaptic plasticity and memory formation in nitric oxide synthase inhibitor-treated rats. Proc. Natl. Acad. Sci. 90: 9191-9194.

Bredt, D.S. and Snyder, S.H. 1992. Nitric oxide, a novel neuronal messenger. Neuron 8: 3-11.

Burghardt, N.S., Sullivan, G.M., McEwen, B.S., Gorman, J.M., and LeDoux, J.E. 2004. The selective serotonin reuptake inhibitor citalopram increases fear after acute treatment but reduces fear with chronic treatment: A comparison with tianeptine. Biol. Psychiatry 55: $1171-1178$.

Chapman, P.F., Kariss, E.W., Keenan, C.L., and Brown, T.H. 1990. Long-term synaptic potentiation in the amygdala. Synapse 6: $271-278$.

Chapman, P.F., Atkins, C.M., Allen, M.T., Haley, J.E., and Steinmetz, J.E. 1992. Inhibition of nitric oxide synthesis impairs two different forms of learning. Neuroreport 3: 567-570.

Chien, W.L., Liang, K.C., Teng, C.M., Kuo, S.C., Lee, F.Y., and Fu, W.M. 2003. Enhancement of long-term potentiation by a potent nitric oxide-guanylyl cyclase activator,

3-(5-hydroxymethyl-2-furyl)-1-benzyl-indazole. Mol. Pharmacol. 63: $1322-1328$.

Davis, M., Redmond Jr., D.E., and Baraban, J.M. 1979. Noradrenergic agonists and antagonists: Effects on conditioned fear as measured by the potentiated startle paradigm. Psychopharmacology 65: 111-118.

Denninger, J.W. and Marletta, M.A. 1999. Guanylate cyclase and the NO/cGMP signaling pathway. Biochim. Biophys. Acta 1411: 334-350.

Doyle, C., Holscher, C., Rowan, M.J., and Anwyl, R. 1996. The selective neuronal NO synthase inhibitor 7-nitro-indazole blocks both long-term potentiation and depotentiation of field EPSPs in rat hippocampal CA1 in vivo. J. Neurosci. 16: 418-424.

Hall, K.U., Collins, S.P., Gamm, D.M., Massa, E., DePaoli-Roach, A.A., and Uhler, M.D. 1998. Phosphorylation-dependent inhibition of protein phosphatase-1 by G-substrate. J. Biol. Chem. 274: 3485-3495.

Hawkins, R.D., Kandel, E.R., and Siegelbaum, S.A. 1993. Learning to modulate transmitter release: Themes and variations in synaptic plasticity. Annu. Rev. Neurosci. 16: 625-665.

Hawkins, R.D., Son, H., and Arancio, O. 1998. Nitric oxide as a retrograde messenger during long-term potentiation in hippocampus. Prog. Brain Res. 118: 155-172.

Holscher, C., McGlinchey, L., Anwyl, R., and Rowan, M.J. 1996. 7-Nitroindazole, a selective neuronal nitric oxide synthase inhibitor in vivo, impairs spatial learning in the rat. Learn. Mem. 2: 267-278.

Hood, J. and Granger, H.J. 1998. Protein kinase G mediates vascular endothelial growth factor-induced Raf-1 activation and proliferation in human endothelial cells. J. Biol. Chem. 273: 23504-23508.

Huang, Y.Y. and Kandel, E.R. 1998. Postsynaptic induction and PKA-dependent expression of LTP in the lateral amygdala. Neuron 21: $169-178$

Huang, Y.Y., Martin, K.C., and Kandel, E.R. 2000. Both protein kinase A and mitogen-activated protein kinase are required in the amygdala for the macromolecular synthesis-dependent late phase of long-term potentiation. J. Neurosci. 20: 6317-6325.

Josselyn, S.A., Shi, C., Carlezon Jr., W.A., Neve, R.L., Nestler, E.J., and Davis, M. 2001. Long-term memory is facilitated by CAMP response element binding protein overexpression in the amygdala. J. Neurosci. 21: $2404-2412$.

Ko, G.Y. and Kelly, P.T. 1999. Nitric oxide acts as a postsynaptic signaling molecule in calcium/calmodulin-induced synaptic potentiation in hippocampal CA1 pyramidal neurons. J. Neurosci. 19: $6784-6794$.

Li, X.F., Stutzmann, G.E., and LeDoux, J.E. 1996. Convergent but temporally separated inputs to lateral amygdala neurons from the auditory thalamus and auditory cortex use different postsynaptic receptors: In vivo intracellular and extracellular recordings in fear conditioning pathways. Learn. Mem. 3: 229-242.

Lu, Y.F., Kandel, E.R., and Hawkins, R.D. 1999. Nitric oxide signaling contributes to late-phase LTP and CREB phosphorylation in the hippocampus. J. Neurosci. 19: 10250-10261.

McKernan, M.G. and Shinnick-Gallagher, P. 1997. Fear conditioning induces a lasting potentiation of synaptic currents in vitro. Nature 390: $607-611$.

Monzón, M.E., Varas, M.M., and De Barioglio, S.R. 2001. Anxiogenesis induced by nitric oxide synthase inhibition and anxiolytic effect of melanin-concentrating hormone $(\mathrm{MCH})$ in rat brain. Peptides 22: 1043-1047.

O'Dell, T.J., Huang, P.L., Dawson, T.M., Dinerman, J.L., Snyder, S.H., Kandel, E.R., and Fishman, M.C. 1994. Endothelial NOS and the blockade of LTP by NOS inhibitors in mice lacking neuronal NOS. Science 265: 542-546.

Paxinos, G. and Watson, C. 1997. The rat brain in stereotaxic coordinates. Academic Press, San Diego, CA.

Schafe, G.E. and LeDoux, J.E. 2000. Memory consolidation of auditory Pavlovian fear conditioning requires protein synthesis and protein kinase A in the amygdala. J. Neurosci. 20: 1-5.

Schafe, G.E., Atkins, C.M., Swank, M.W., Bauer, E.P., Sweatt, J.D., and LeDoux, J.E. 2000. Activation of ERK/MAP Kinase in the amygdala is required for memory consolidation in Pavlovian fear conditioning. $J$. Neurosci. 20: $8177-8187$.

Schafe, G.E., Nader, K., Blair, H.T., and LeDoux, J.E. 2001. Memory consolidation of Pavlovian fear conditioning: A cellular and molecular perspective. Trends Neurosci. 24: 540-546.

Schafe, G.E., Bauer, E.P., Rosis, S., Farb, C.R., Rodrigues, S.M., and LeDoux, J.E. 2005a. Memory consolidation of Pavlovian fear conditioning requires nitric oxide signaling in the lateral amygdala. Eur. J. Neurosci. 22: 201-211.

Schafe, G.E., Doyere, V., and LeDoux, J.E. 2005b. Tracking the fear engram: The lateral amygdala is an essential locus of fear memory storage. J. Neurosci. 25: 10010-10014.

Schuman, E.M. and Madison, D.V. 1991. A requirement for the intercellular messenger nitric oxide in long-term potentiation. Science 254: 1503-1506.

Son, H., Hawkins, R.D., Martin, K., Kiebler, M., Huang, P.L., Fishman, M.C., and Kandel, E.R. 1996. Long-term potentiation is reduced in mice that are doubly mutant in endothelial and neuronal nitric oxide synthase. Cell 87: 1015-1023.

Son, H., Lu, Y.F., Zhuo, M., Arancio, O., Kandel, E.R., and Hawkins, R.D. 1998. The specific role of cGMP in hippocampal LTP. Learn. Mem. 5: $231-245$.

Sullivan, G.M., Apergis, J., Gorman, J.M., and LeDoux, J.E. 2003. Rodent doxapram model of panic: Behavioral effects and c-Fos immunoreactivity in the amygdala. Biol. Psychiatry 53: $863-870$.

Suzuki, Y., Ikari, H., Hayashi, T., and Iguchi, A. 1996. Central administration of a nitric oxide synthase inhibitor impairs spatial memory in spontaneous hypertensive rats. Neurosci. Lett. 207: 105-108.

Tsvetkov, E., Carlezon, W.A., Benes, F.M., Kandel, E.R., and Bolshakov, V.Y. 2002. Fear conditioning occludes LTP-induced presynaptic enhancement of synaptic transmission in the cortical pathway to the lateral amygdala. Neuron 34: 289-300.

Watanabe, Y., Saito, H., and Abe, K. 1995. Nitric oxide is involved in long-term potentiation in the medial but not lateral amygdala synapses in vitro. Brain Res. 688: 233-236.

Weisskopf, M.G., Bauer, E.P., and LeDoux, J.E. 1999. L-type voltage-gated calcium channels mediate NMDA-independent associative long-term potentiation at thalamic input synapses to the amygdala. J. Neurosci. 19: 10512-10519.

Zhuo, M., Hu, Y., Schultz, C., Kandel, E.R., and Hawkins, R.D. 1994. Role of guanylyl cyclase and cGMP-dependent protein kinase in long-term potentiation. Nature 368: 635-639.

Zou, L.B., Yamada, K., Tanaka, T., Kameyama, T., and Nabeshima, T. 1998. Nitric oxide synthase inhibitors impair reference memory formation in a radial arm maze task in rats. Neuropharmacology 37: 323-330.

Received June 19, 2008; accepted in revised form August 11, 2008. 


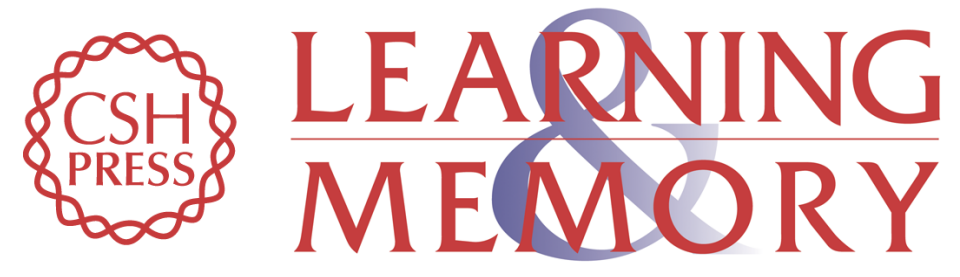

\section{The NO-cGMP-PKG signaling pathway regulates synaptic plasticity and fear memory consolidation in the lateral amygdala via activation of ERK/MAP kinase}

Kristie T. Ota, Vicki J. Pierre, Jonathan E. Ploski, et al.

Learn. Mem. 2008, 15:

Access the most recent version at doi: $10.1101 / \mathrm{lm} .1114808$

References This article cites 46 articles, 20 of which can be accessed free at: http://learnmem.cshlp.org/content/15/10/792.full.html\#ref-list-1

License

Email Alerting Service

Receive free email alerts when new articles cite this article - sign up in the box at the top right corner of the article or click here. 